\title{
The effect of wavy leading edges on aerofoil-gust interaction noise
}

\author{
Alex S.H. Lau, Sina Haeri, Jae Wook Kim* \\ Aerodynamics and Flight Mechanics Research Group, Aeronautics and Astronautics, University of Southampton, \\ SO17 1BJ, United Kingdom
}

\section{A R T I C L E I N F O}

\section{Article history:}

Received 18 February 2013

Received in revised form

23 June 2013

Accepted 26 June 2013

Handling Editor: R.E. Musafir

Available online 17 August 2013

\begin{abstract}
A B S T R A C T
High-order accurate numerical simulations are performed to investigate the effects of wavy leading edges (WLEs) on aerofoil-gust interaction (AGI) noise. The present study is based on periodic velocity disturbances predominantly in streamwise and vertical directions that are mainly responsible for the surface pressure fluctuation of an aerofoil. In general, the present results show that WLEs lead to reduced AGI noise. It is found that the ratio of the wavy leading-edge peak-to-peak amplitude (LEA) to the longitudinal wavelength of the incident gust $\left(\lambda_{g}\right)$ is the most important factor for the reduction of AGI noise. It is observed that there exists a tendency that the reduction of AGI noise increases with LEA $/ \lambda_{g}$ and the noise reduction is significant for LEA $/ \lambda_{g} \geq 0.3$. The present results also suggest that any two different cases with the same LEA/ $\lambda_{g}$ lead to a strong similarity in their profiles of noise reduction relative to the straight leading-edge case. The wavelength of wavy leading edges (LEW), however, shows minor influence on the reduction of AGI noise under the present gust profiles used. Nevertheless, the present results show that a meaningful improvement in noise reduction may be achieved when $1.0 \leq \mathrm{LEW} / \lambda_{\mathrm{g}} \leq 1.5$. In addition, it is found that the beneficial effects of WLEs are maintained for various flow incidence angles and aerofoil thicknesses. Also, the WLEs remain effective for gust profiles containing multiple frequency components. It is discovered in this paper that WLEs result in incoherent response time to the incident gust across the span, which results in a decreased level of surface pressure fluctuations, hence a reduced level of AGI noise.
\end{abstract}

(c) 2013 Elsevier Ltd. All rights reserved.

\section{Introduction}

Aerodynamic sound is generated when a solid surface, such as a wind-turbine blade, is situated in an incident flow which is unsteady and non-uniform. Noise generated in this manner will be referred to as aerofoil-gust interaction (AGI) noise herein. The generation of AGI noise is mainly an inviscid phenomenon, where pressure fluctuations are generated to balance the momentum fluctuations that occur due to the distortion of the velocity disturbance, or gust, by the potential flow near the obstacle. AGI noise is an important noise source for many applications involving aerofoils (rotor blades, turbofans, aircraft high-lift devices, etc.) situated in impinging flow disturbances. In recent years, wind-turbine blade noise has gained significant attention due to the expansion of wind farms as a sustainable source of energy. They are often operating against atmospheric wind gusts and turbulent wakes from upstream wind turbines, which are potential sources of AGI noise.

\footnotetext{
* Corresponding author. Tel.: +44 238059 4886; fax: +44 2380593058.

E-mail address: j.w.kim@soton.ac.uk (J.W. Kim).
} 
Migliore and Oerlemans [1] showed that AGI noise becomes dominant over trailing-edge noise when there is a moderate level of disturbances in the incident flow. Therefore, a more in-depth study of AGI noise and its reduction is required.

Wind-turbine noise has been investigated theoretically. For instance, a theoretical approach particularly for the study of AGI noise was developed by Goldstein [2] utilizing the rapid distortion theory. By assuming the flow to be inviscid and nonheat-conducting, he linearized the governing equations and rewrote them into a linear inhomogeneous equation in terms of a perturbation potential. For a compressible flow, the inhomogeneous wave equation derived by Goldstein has variable coefficients and functions within the source term and boundary conditions. Hence the equation has to be solved numerically. Myers and Kerschen [3,4] developed approximate close-form expressions of the equation. They considered a 2D irrotational compressible mean flow, which was assumed to be a small perturbation to a uniform flow. Superposition of small amplitude harmonic components was used to represent the upstream divergence-free vortical velocity. They further assumed small flow incidence angle and high frequency of converted disturbances. With these assumptions, a simplified form of Goldstein's equation was derived. Flat plate aerofoils with and without camber have been analysed by Myers and Kerschen [3,4]. Evers and Peake [5] derived an extended equation applicable to an aerofoil with small but non-zero thickness, camber and angle of attack in transonic mean flows. The theoretical models provide useful ground for the study of AGI noise in two-dimensional (2D) domain.

There also exist semi-empirical approaches to predict the AGI noise, which were developed based on the results of previous experimental and theoretical studies. For instance, Lowson [6] combined the AGI theory of Amiet [7], which considers the response of an infinitely thin flat plate, with a semi-empirical gust field to predict the wind turbine noise. The parameters of the semi-empirical model were chosen to reflect the test conditions of a certain set of experimental data. The major advantage of semi-empirical models is that they are computationally inexpensive, easy to use, and fast to return results. However, the validity of using these models in various flow conditions is questionable since they are restricted by many constraints.

Computational aeroacoustics (CAA) has made significant advancement in recent years to allow for high-order accurate simulations of aerofoil noise source mechanisms. However, the computational cost needed to cover the whole range of wind-turbine noise sources accurately and in reasonable time remains prohibitively high. Hence, it is sensible to study each type of noise generation mechanism separately. Aerofoil self-noise, which originates within the boundary layer and the wake regions, is avoided in the current simulations by using Euler equations. There exists some fundamental work done on AGI noise based on CAA approaches and they were mostly limited in 2D domain [8-11]. Recent three-dimensional (3D) simulations were performed by Atassi et al. [12] and Hixon et al. [13]. The current research employs an accurate and efficient high-order method [14] for direct computation of AGI noise in 3D domain.

The aim of the current study is to investigate the effect of aerofoil leading-edge geometries on AGI noise. It is well known that the silent flight of owls is attributed to their tiny saw-tooth-shaped serrations on the leading edge of the main flight feathers [15,16]. Soderman [15] conducted experiments using rotor blades with saw-tooth serrations (0.1-0.5 cm in height) attached to the lower surface of the leading edge. He found that the serrated leading edge is effective in reducing high-frequency noise at low tip speeds between 48 and $135 \mathrm{~m} / \mathrm{s}$. However, the aerodynamic effect was highly sensitive to the attachment location of the serrations. Small deviation from the ideal location could lead to overall degradation in aerodynamic performance. A more recent experimental study on owl wings by Ito [16] reported that the post-stall aerodynamic benefit brought about by serrated leading edges was only observed at low Reynolds number of $2.1 \times 10^{4}$.

Another type of leading-edge geometry can be found in the pectoral flippers of humpback whales, which have tubercles. Several studies showed that leading-edge tubercles may lead to a more gradual stall and better post-stall performance than straight leading edges at the expense of marginal reduction in pre-stall performance [17-25]. The aerodynamic benefits of leading-edge tubercles have been observed over a wide range of Reynolds numbers. While most of the work has focused on the aerodynamic aspects, Hansen et al. [26] recently studied experimentally the aeroacoustic effect (tonal noise) and found that leading-edge tubercles, particularly those with the smallest wavelength and the largest amplitude tested, reduced the tonal noise significantly.

In light of these studies, the authors investigate a spanwise sinusoidal profile of leading edges, which is referred to as wavy leading edges WLEs herein. Unlike the aforementioned serrations and tubercles, the proposed WLEs preserve the same aerofoil section and planform area with those of the original straight leading-edge counterparts. It was recently shown that WLEs produce similar aerodynamic benefits that were obtained by using the tubercles [25]. It is envisaged that WLEs may also be beneficial in reducing AGI noise in the presence of incident gusts, which has not been extensively investigated to this date. In this study, the authors consider periodic velocity disturbances predominantly in streamwise and vertical directions (with insignificant variation in the spanwise velocity) since they are the main components causing pressure fluctuations on the aerofoil surface. The present study aims to deliver a fundamental understanding of the effects of WLEs on AGI noise by performing high-fidelity numerical simulations.

The organization of the paper is as follows. Sections 2 and 3 introduce the present numerical methodology used and its validation for AGI noise calculations. In Section 4, parametric studies are performed to identify the effects of peak-to-peak amplitude and wavelength of WLEs. In Section 5, the noise reduction mechanism of WLEs is explained. The effects of WLEs are further demonstrated in Section 6 for various flow incidence angles and aerofoil thicknesses. In Section 7, multimode gusts with more than one constituent frequency components are employed to test the effectiveness of WLEs. Finally, concluding remarks are given in Section 8. 


\section{The current numerical methodology}

The governing equations used for the current study are the Euler equations, which can be written in the conservationform as a compact vector equation

$$
\frac{\partial \mathbf{Q}}{\partial t}+\frac{\partial \mathbf{E}}{\partial x}+\frac{\partial \mathbf{F}}{\partial y}+\frac{\partial \mathbf{G}}{\partial z}=0
$$

where $\mathbf{Q}=(\rho, \rho u, \rho v, \rho w, \rho e)^{T}$ is the 3D conservative flow variables vector, with $\rho, u, v, w$ and $e$ the density, $x$-velocity component, $y$-velocity component, $z$-velocity component and total energy per unit mass, respectively; $t$ is time; E, F, G are the inviscid flux terms, which are functions of $\mathbf{Q}$, such that

$$
\begin{gathered}
\mathbf{E}=\left[\rho u, \rho u^{2}+p, \rho u v, \rho u w,(\rho e+p) u\right]^{T}, \\
\mathbf{F}=\left[\rho v, \rho u v, \rho v^{2}+p, \rho v w,(\rho e+p) v\right]^{T}, \\
\mathbf{G}=\left[\rho w, \rho u w, \rho v w, \rho w^{2}+p,(\rho e+p) w\right]^{T},
\end{gathered}
$$

where $p$ is the pressure. Since the flow considered is inviscid, the aerofoil self-noise that originates within the boundary layer and the wake regions cannot develop.

The efficiency and accuracy of the current numerical methodology has been demonstrated in a previous study [14] concerning 2D problems. One of the unique features of the methodology is a sponge-zone technique that allows for suppressing wave reflections as well as embedding desired gust flows through the outer boundaries [14]. This new sponge $\mathrm{BC}$ forcing term can be represented by

$$
\mathbf{S}_{\mathrm{new}}=\sigma\left(\begin{array}{c}
\rho-\rho_{\infty} \\
W\left(\rho u-\rho_{\infty} u_{\text {gust }}\right) \\
W\left(\rho v-\rho_{\infty} v_{\text {gust }}\right) \\
W\left(\rho w-\rho_{\infty} w_{\text {gust }}\right) \\
p-p_{\infty}
\end{array}\right)
$$

with

$$
W= \begin{cases}W(x) & \text { for } x_{\min } \leq x \leq x_{\min }+\phi L_{d} \\ 0 & \text { otherwise }\end{cases}
$$

where

$$
W(x)=\frac{1}{2}\left\{\cos \left[\frac{\pi\left(x-x_{\min }\right)}{\phi L_{d}}\right]+1\right\}
$$

$x_{\min }$ is the minimum $x$ at which the inflow boundary is situated, $L_{d}$ is the length of the domain along the $x$ direction, $\phi$ is a free parameter and $0 \leq \phi \leq 1$. The blending factor $\sigma$ smoothly varies on the thickness of the sponge zone $L_{S}$, from zero in the physical domain to some positive value $\sigma_{0}$ at the outer boundaries of the sponge zone, where non-reflecting characteristic $\mathrm{BC}[27,28]$ are applied. As a summary, the new sponge $\mathrm{BC}$ requires three free parameters: the maximum damping coefficient $\sigma_{0}$, the sponge zone thickness $L_{S}$ and the velocity forcing length ratio parameter $\phi$ which were determined in the previous study [14].

For the spatial discretization, fourth-order optimized compact finite difference (FD) schemes [29] are used. Sixth-order optimized compact low-pass filters developed by Kim [30] are used to ensure numerical stability. All the filter coefficients are functions of the cut-off wavenumber $\kappa_{C}$ only, and can be adjusted easily for different problems. Since the boundary FD schemes are non-central, the solution resolution at the boundary points is lower. Kim [30] suggested a weighting factor $\epsilon$, which reduces the cut-off wavenumber $\kappa_{C}$ to lower values $\kappa_{C i}$ for the boundary points. Kim suggested $\epsilon=0.085$ as the optimal value for the current schemes. This optimal value, which corresponds to the stability range $0.5 \pi \leq \kappa_{C} \leq 0.88 \pi$, is used in the current study. For parallel calculations, a scalable asynchronous algorithm similar to the one suggested in [31] is implemented for the MPI communications. In addition, compact FD schemes with extended stencils containing three halo points near additional sub-domain interfaces are used as suggested by Kim and Sandberg [32]. Kim and Sandberg suggested a smooth profile of the cut-off wavenumber around the interfaces. The wavenumber reduces from $\kappa_{C}$ at the interior points smoothly to some value close to $\kappa_{C}^{*}$ at the interface points. Hence, there are three input parameters to the compact discrete filters used in the current study. They are the cut-off wavenumbers $\kappa_{C}$ and $\kappa_{C}^{*}$, and the boundary weighting factor $\epsilon$.

For time integration, a standard fourth-order four-stage Runge-Kutta time marching scheme is used to advance the solution in time in the current study.

\section{The validation of the current methodology}

The present validation consists of 2D flat-plate calculations compared with theoretical results and 3D calculations of an aerofoil with WLEs verified by a grid-convergence test, a domain-size test and parametric studies. Numerical input variables used for all of the present calculations are shown in Table 1. $L$ denotes the mean chord length. The filter input parameters 
Table 1

The constant set of input parameters.

\begin{tabular}{lllllll}
\hline$\kappa_{C}$ & $\kappa_{C}^{*}$ & $\epsilon$ & $\sigma_{0}$ & $L_{S}$ & $\phi$ & CFL \\
\hline $0.87 \pi$ & $0.80 \pi$ & 0.085 & 4.0 & $3 L$ & 1.0 \\
\hline
\end{tabular}

are based on the studies by Kim [30] and Kim and Sandberg [32]; and the sponge BC parameters are determined by the same procedure as shown in [14].

\subsection{Validation against theoretical results}

In the first validation study, the theoretical prediction of far-field pressure for a flat plate aerofoil at zero angle of attack proposed by Myers and Kerschen [3] is used for comparison. Their theoretical far-field pressure is denoted by $P_{\mathrm{MK}}$, and is plotted as $\left|P_{\mathrm{MK}}\right| \sqrt{r_{\mathrm{ph}}}$, where $r_{\mathrm{ph}}$ is the physical radial distance from the leading edge. To compare with the theoretical results, the current numerical results to be plotted are $P_{\mathrm{RMS}} \sqrt{r_{\mathrm{ph}}} / \zeta$, where $P_{\mathrm{RMS}}$ is the root mean square pressure perturbation and $\zeta$ is the perturbed velocity magnitude and is a small dimensionless number $-\zeta=0.01$ is used in this paper. $P_{\mathrm{RMS}}$ is defined as

$$
P_{\text {RMS }}=\sqrt{\left[\sum_{n=1}^{N}\left(p^{\prime 2}\right) d t_{n}\right] / t_{\text {sample }}},
$$

where $p^{\prime}$ is the pressure perturbation about the mean value; $d t_{n}$ is the time step size at the $n$th time step; $t_{\text {sample }}$ is the sampling time duration.

The initial numerical tests are carried out based on a flat plate with zero thickness at zero angle of attack in a mean flow with a perturbed velocity defined as

$$
u_{i}^{\prime}=\left(-1^{(i-1)}\right)\left\{A_{\mathrm{mp}} \cos \left[k\left(x+y-M_{\infty} t\right)\right]+A_{\mathrm{mp}} \sin \left[k\left(x+y-M_{\infty} t\right)\right]\right\},
$$

which is similar to that used by Myers and Kerschen [3]. In Eq. (3), $i=1$ and 2 denote the $x$ - and $y$-direction coordinates, respectively; $M_{\infty}$ is the freestream Mach number; $A_{\mathrm{mp}}$ is the amplitude parameter; and, $k$ is the reduced frequency. It should however be noted that $k=2 k_{\mathrm{MK}}$, where $k_{\mathrm{MK}}$ is the reduced frequency defined in the Myers and Kerschen theory [3]. This is due to the fact that the full chord length of the flat plate is used as the reference length scale here, whereas in [3] the semichord was used as the reference. The grid around the flat plate aerofoil consists of six blocks, which are arranged in a $H$-topology as shown in an earlier publication [14].

The first observation is on the effect of the reduced frequency $k$, which varies from three to ten with an increment of one. The free-stream Mach number $M_{\infty}$ remains constant at 0.5 for this. The resulting directivity patterns for the $k$ tests are shown in Figs. 1 and 2. The second observation is on the effect of $M_{\infty}$, which varies from 0.45 to 0.65 with an increment of 0.05 . $k$ is kept at 4 for this. The results for the $M_{\infty}$ test are shown in Figs. 3 and 4 . It can be seen that the numerical and the theoretical results show very good agreement with each other in general. An additional test with $k=12$ and $M_{\infty}=0.6$ is performed for further validation at a high gust frequency. This is equivalent to the case presented in Fig. 4a of Myers and Kerschen [3] $\left(k_{\mathrm{MK}}=6\right)$. Fig. 5 shows the result of the additional comparison between the present simulation result and the prediction of the theory (plotted by a digitized version of Fig. 4a from [3] as well as our own re-production of the theory). This shows that the present methodology is suitable and accurate for this type of simulations in a wide range of gust frequencies and free-stream Mach numbers. The accuracy and efficiency of the current methodology have also been demonstrated in a previous study [14], in which some published solutions to the category 3 problem 1 from the Third [33] and Fourth [34] CAA Workshops were used for comparison.

\subsection{Validation against experimental data}

To further validate the code, lift coefficient of the aerofoil is calculated and compared with the experimental measurements provided by Sheldahl and Klimas [35] for a NACA0015 aerofoil with a straight leading edge and no incident gust at $R e=3.6 \times 10^{5}$. Since the current numerical simulations are inviscid, no meaningful data for drag can be drawn. Only the lift at low angles of attack is computed and compared with the measured data for validation purpose. While grid convergence studies are performed and are discussed in Section 3.3, it is noteworthy that the baseline grid (see Table 2) is used in this section. The lift is calculated by integrating all the elemental surface pressure force components around the 3D aerofoil in the vertical direction. We have tested the following free-stream Mach numbers: 0.1, 0.3 and 0.5 . The values of lift coefficient $\left(C_{L}\right)$ are calculated for $\alpha=-1^{\circ}, 0^{\circ}, 1^{\circ}, 2^{\circ}, 3^{\circ}$. The computed lift coefficients are presented in Fig. 6, where the left figure is directly from the current calculation and the right one shows the current data multiplied by the Prandtl-Glauert factor $\sqrt{1-M_{\infty}^{2}}$ that converts the current compressible $C_{L}$ to its corresponding incompressible value. It can be seen from the plot on the left that as Mach number increases, the slope of the current $C_{L}-$ to- $\alpha$ lines also increases. This increase in slope is due to the compressibility effect as suggested by the plot on the right, which shows that the current $C_{L}-$ to- $\alpha$ lines 

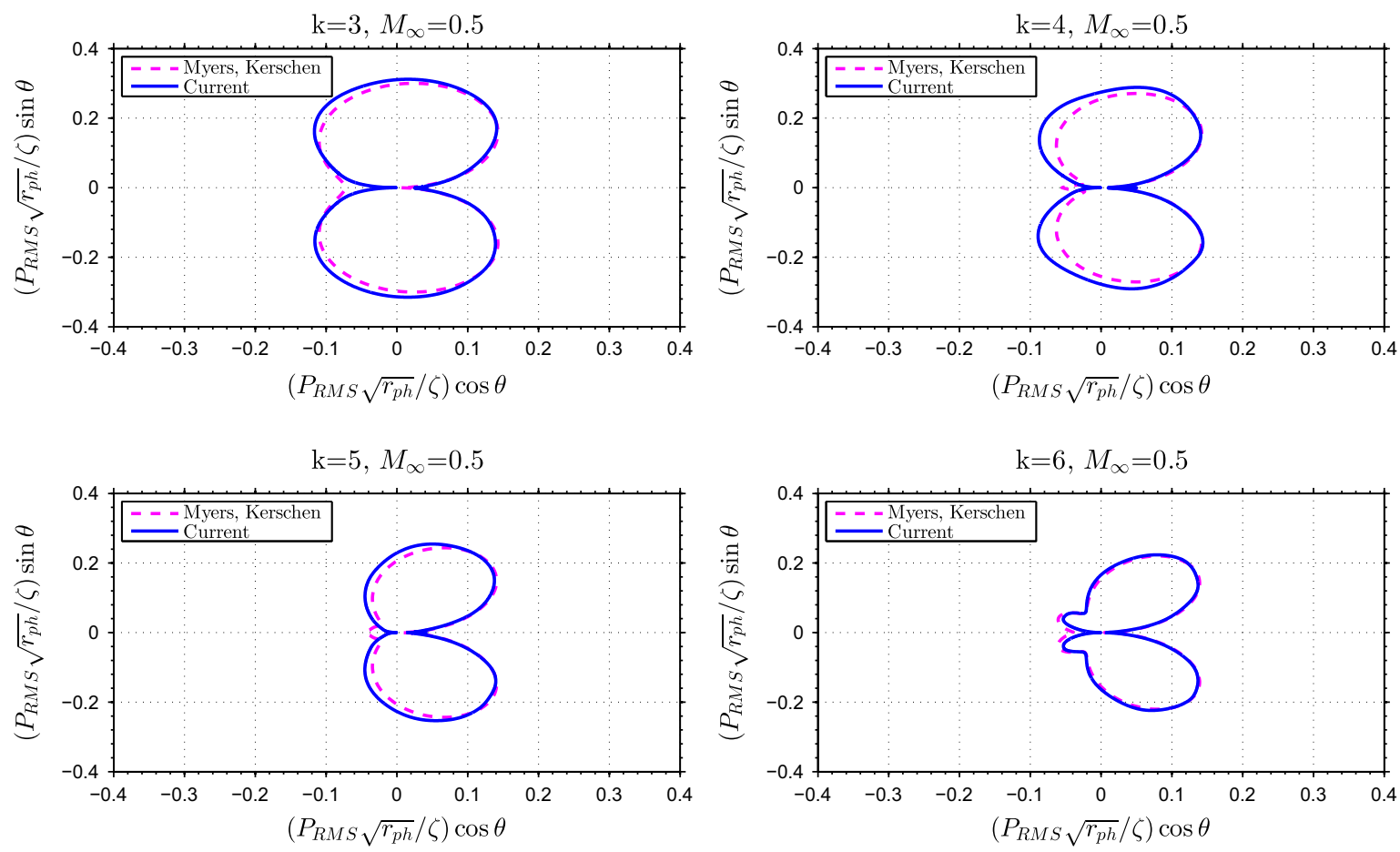

Fig. 1. The effect of reduced frequency, $k=3,4,5$ and 6 .
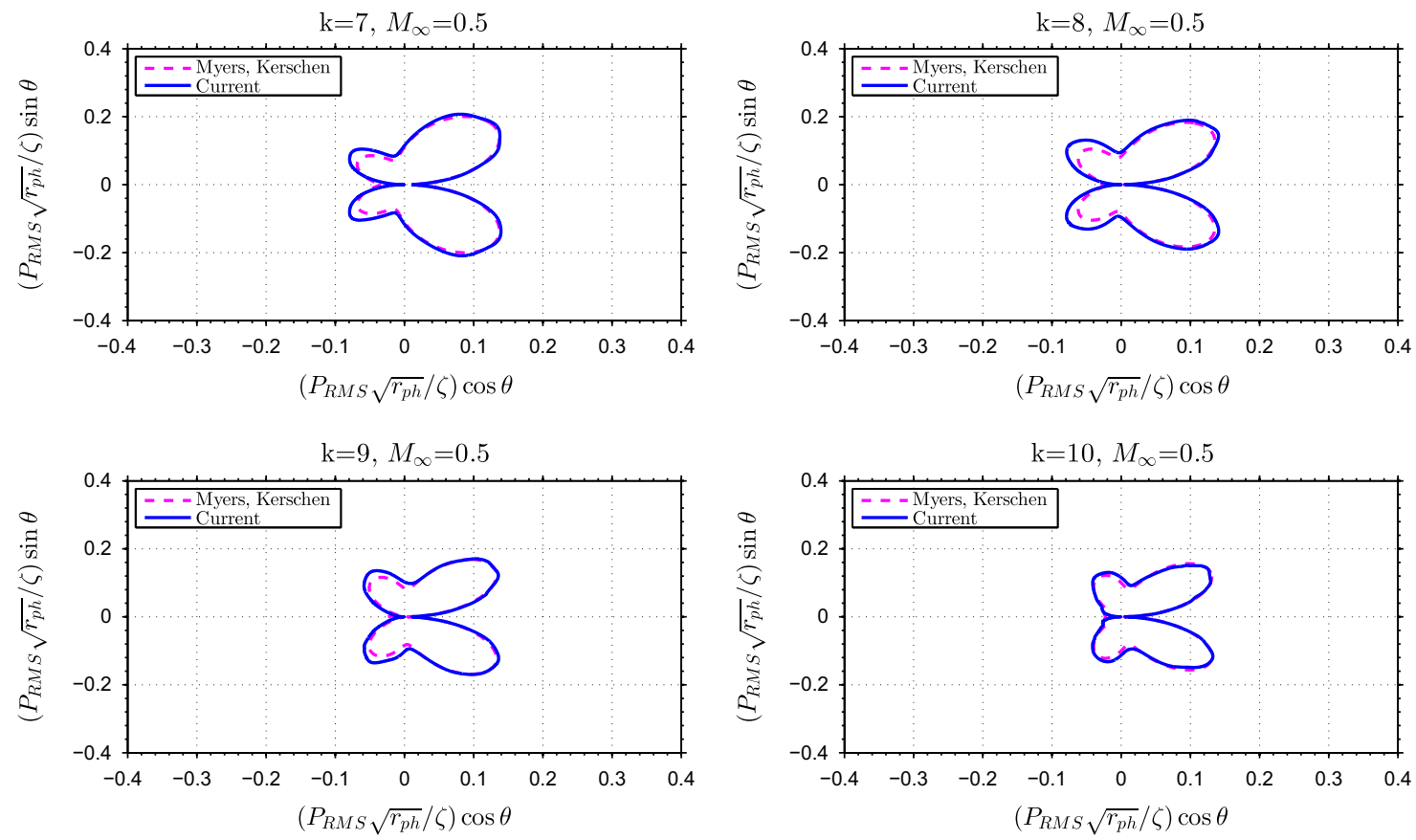

Fig. 2. The effect of reduced frequency, $k=7,8,9$ and 10 .

at different Mach numbers collapse onto a single line after applying the conversion factor. The discrepancy between the current and the experimental data is conjectured to stem from the omission of viscosity in the current simulation.

\subsection{The effect of WLEs on aerodynamic performance}

In this section a grid convergence study is performed first to identify the optimum baseline grid for the aerofoil simulations with WLEs and then the effects of the WLEs on the lift coefficient are studied. Fig. 7 shows a typical of the 



Fig. 3. The effect of free-stream Mach number, $M_{\infty}=0.4,0.45,0.5$ and 0.55 .


Fig. 4. The effect of free-stream Mach number, $M_{\infty}=0.6$ and 0.65

present aerofoil geometry and the frame of computational domain used in this work, where the definitions of leading-edge amplitude and wavelength, denoted by LEA and LEW, respectively, are given. Note that LEA is peak-to-peak based.

The imposed perturbed velocity components are given by

$$
u_{i}^{\prime}=A_{i} \cos \left(k_{1} x+k_{2} y+\omega t\right)+B_{i} \sin \left(k_{1} x+k_{2} y+\omega t\right) \quad \text { for } i=1,2
$$

where $A_{1}=B_{1}=0.005$ and $A_{2}=B_{2}=-0.004$ (parameters to set the amplitude of the perturbation) are constantly used in this paper. The prescribed velocity disturbances are mainly in the streamwise and vertical directions, whereas the spanwise component is constrained to satisfy the divergence-free condition, i.e. $\partial u^{\prime}{ }_{i} / \partial x_{i}=0$, to prevent the generation of unwanted pressure fluctuations in the free stream. The streamwise and vertical components are the major cause of pressure fluctuations on the aerofoil surface leading to AGI (aerofoil-gust interaction) noise. The free-stream Mach number is set to $M_{\infty}=0.5$ for the rest of the paper unless otherwise specified. The indices $i=1$ and 2 denote the Cartesian coordinates; $u^{\prime}{ }_{i}$ represents the velocity perturbation along the $i$ th direction; $k_{1}$ and $k_{2}$ are the non-dimensional wavenumbers; and, $\omega=-k_{1} M_{\infty}$ is the angular frequency for a wave travelling in the positive $x$-direction (from left to right).

For the grid convergence tests, two grids, one with double the grid density of the other, are used. Then, two different sizes of the domain in span (with periodic boundary conditions) are tested. Two grids of the same cell density on the $x-y$ plane, one with two LEWs in span and the other with only one, are used. For these two tests, $k_{1}=k_{2}=2 \pi$ and $\alpha=0$ are used. The cross-section of the aerofoil used is uniformly based on NACA0015. For both tests, LEA $=0.1 \mathrm{~L}$ and $\mathrm{LEW}=0.5 \mathrm{~L}$. The numerical results presented are directivity patterns of $P_{\mathrm{MS}}\left(=P_{\mathrm{RMS}}^{2}\right)$ in the $x-y$ plane, which are averaged over the span in $z$-coordinate and obtained at a radial distance $R=4 L$ from the averaged centre of the aerofoil section. 


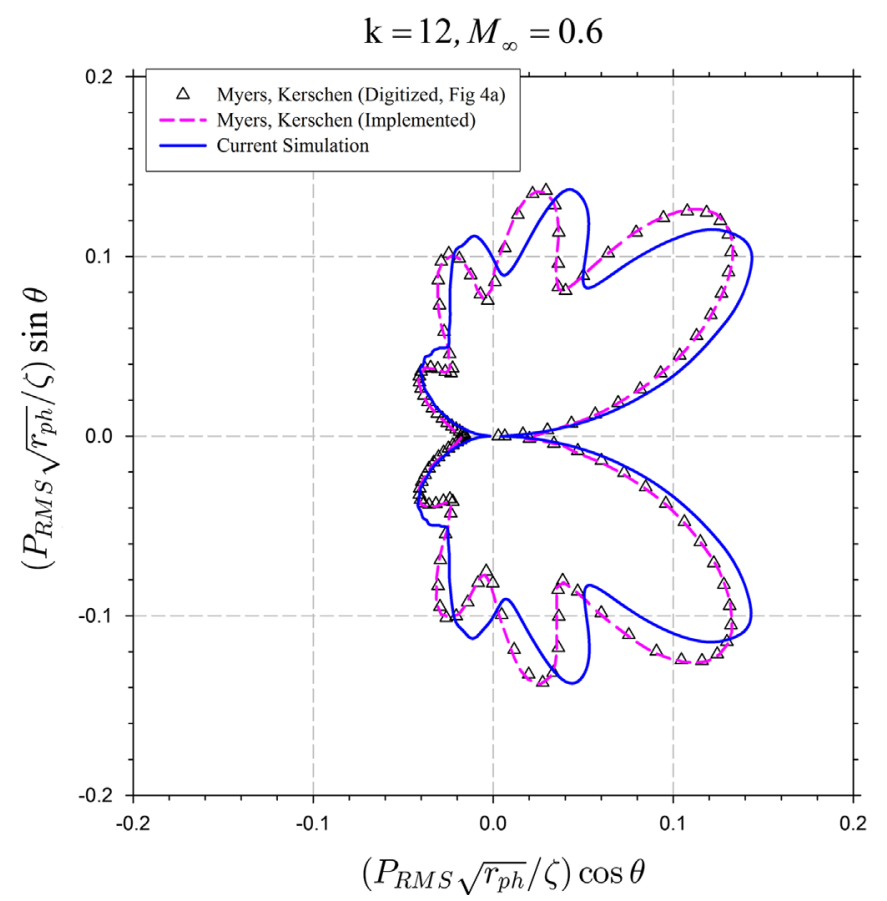

Fig. 5. Validation of the current implementation of the Myers and Kerschen theory [3].

Table 2

The number of grid points used in the baseline grid.

\begin{tabular}{ll}
\hline Up- or down-stream & 131 \\
Above or below & 131 \\
Upper and lower surface of aerofoil & 131 \\
Spanwise & 36 \\
Total & $3,706,776$ \\
\hline
\end{tabular}

NACA0015 $C_{L}$ versus $\alpha$

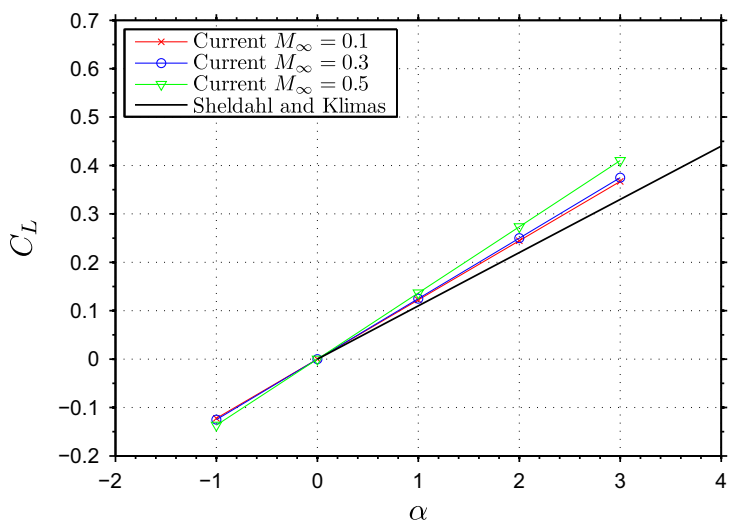

NACA0015 $C_{L}$ versus $\alpha$, Incomressible

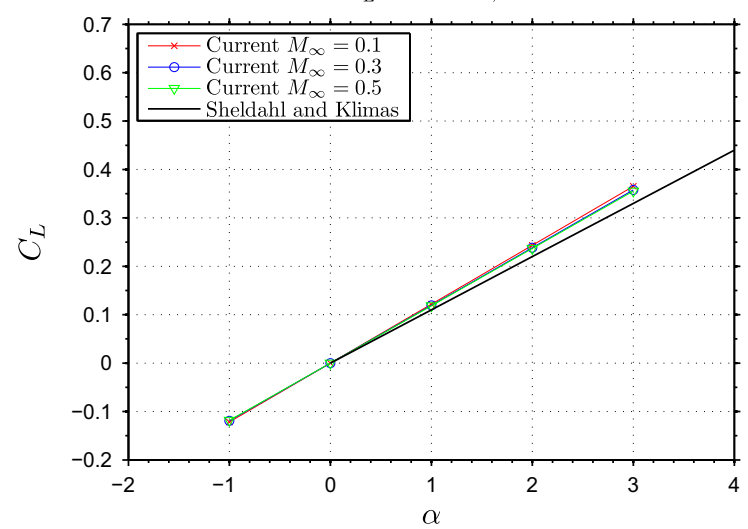

Fig. 6. Calculated lift coefficients (for a straight leading edge) compared with experimental data.

Fig. 8 shows the resulting directivity patterns of $P_{\mathrm{MS}}=P_{\mathrm{RMS}}^{2}$ for the two tests. From the plot on the right of Fig. 8 , it can be seen that the directivity patterns for the two grids of equal grid density but with different span lengths are identical. This indicates that one leading-edge wavelength in span is sufficient for the subsequent investigation. The plot on the left of Fig. 8 shows that the two grids with different grid density yield very little difference in solution. This result provides a baseline grid for simulations in the case of $k_{1}=k_{2}=2 \pi$ as shown in Table 2.

This baseline grid contains about 11 grid points per gust wavelength near the domain boundaries where the grid is the coarsest. The same level of grid density per gust wavelength is maintained in this paper for all the other cases with higherfrequency incident gusts. Note that the spanwise number of grid points varies with different values of LEW. 


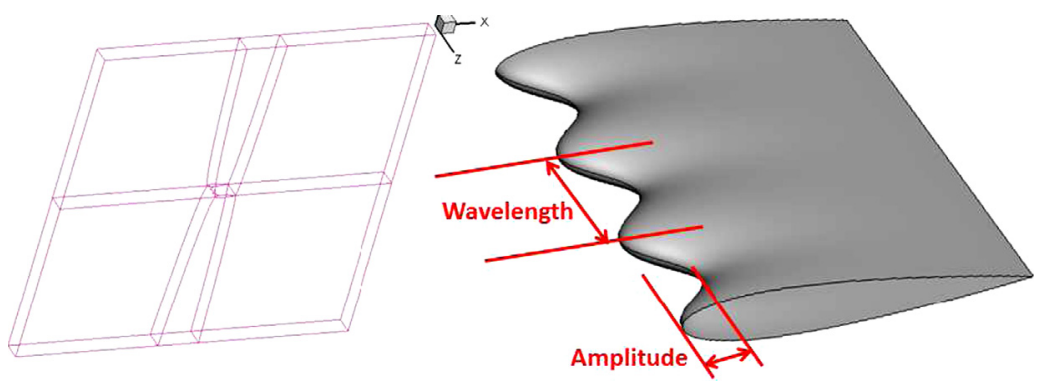

Fig. 7. The grid structure and the definitions of leading-edge amplitude and wavelength.


Fig. 8. Grid convergence and domain size tests.

Table 3

Test cases for $C_{L}$ validation of the 3D aerofoil with WLEs.

\begin{tabular}{llll}
\hline Test case & LEA & LEW & $\alpha$ tested \\
\hline Case 1 & $0.1 L$ & $0.5 L$ & $0^{\circ}, 1^{\circ}, 2^{\circ}, 3^{\circ}$ \\
Case 2 & $0.2 L$ & $1.0 L$ & $0^{\circ}, 2^{\circ}, 4^{\circ}$ \\
Case 3 & $0.3 L$ & $1.0 L$ & $0^{\circ}, 2^{\circ}, 4^{\circ}$ \\
Case 4 & $0.4 L$ & $1.0 L$ & $0^{\circ}, 2^{\circ}$ \\
\hline
\end{tabular}

NACA0015 $C_{\mathrm{L}}$ versus $\alpha$



Fig. 9. Aerodynamic validation of the present 3D aerofoils with WLEs. 
To test the effects of WLEs on the aerodynamic performance we consider an aerofoil with WLE and an incident vortical gust given by Eq. (4), with the same Mach number $\left(M_{\infty}=0.5\right)$ and wavelength $\left(k_{1}=k_{2}=2 \pi\right)$ used in the earlier gridconvergence test. The spanwise size of the domain is set to one LEW as mentioned earlier and periodic boundary conditions are implemented. The test cases are listed in Table 3.

The resulting $C_{L}$ data are plotted in Fig. 9 compared with the data from a straight leading edge without the incident gust. It can be seen that all the calculated data agree with each other very well, which indicates that the averaged aerodynamic performance of the present 3D aerofoils with WLEs is identical to that of the original 2D aerofoil.

Since no viscosity is included in the present study, the result of simulations is meaningful only at low values of $\alpha$. Given the limitation, the validation studies show that the present numerical methodology provides an accurate, reliable and efficient ground for the investigation of AGI noise associated with WLEs.

\section{The effects of wavy leading edge amplitude and wavelength}

In this section, the effects of wavy leading-edge peak-to-peak amplitude (LEA) and wavelength (LEW) with respect to the longitudinal (streamwise) wavelength of the incident gust $\left(\lambda_{g}\right)$ on AGI noise are investigated. Three different gusts with $\lambda_{\mathrm{g}} / L=1.0,0.5$ and $1 / 3$ are used, which are referred to as low-, the medium- and the high-frequency gusts, respectively.

The numerical results are presented in the form of spanwise-averaged $x-y$ plane directivity patterns of the mean-square pressure fluctuation, $P_{\mathrm{MS}}$, representing the sound pressure level, plotted at a radial distance $R=4 L$ from the centre of the aerofoil section. Also, the percentage differences relative to the straight leading-edge case are plotted against the sound propagation angle $\theta$, whose definition is shown in Fig. 10. The test cases in this section are listed in Table 4.

The low-frequency gust is used for Tests 1.0 and 2.0-2.3, and the high- and medium-frequency gusts are used for Tests 1.1 and 3, respectively. The 3D aerofoil section is based on the NACA0015 profile and zero angle of attack is applied in this section. Tests 1.0 and 1.1 investigate the effects of LEA, and Tests 2.0-2.3 and 3 are for the effect of LEW, where the geometric parameters are related with the gust wavelength $\left(\lambda_{g}\right)$. Collectively, these parametric tests should show a glimpse of when the reduction in AGI noise becomes significant.

\subsection{The effects of $L E A$}

The resulting directivity patterns of $P_{\mathrm{MS}}$ from Test 1.0 for the low-frequency gust and the percentage differences relative to the straight leading-edge case are plotted in Fig. 11. It can be seen that the AGI noise level reduces as the peak-to-peak

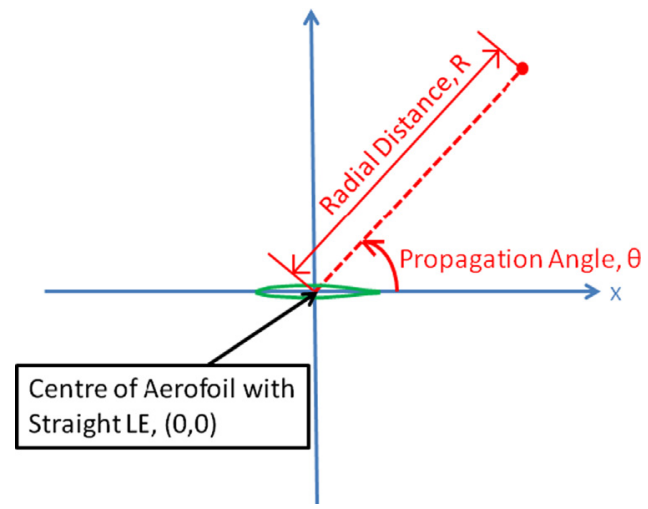

Fig. 10. Definition of sound propagation angle $\theta$.

Table 4

The list of leading-edge and gust parameters tested in this section.

\begin{tabular}{llll}
\hline Test case & LEA & LEW & $\lambda_{g}$ \\
\hline Test 1.0 & $0.1 L$ to $0.5 L^{\mathrm{a}}$ & $1.0 L$ & $1.0 L$ \\
Test 1.1 & $0.1 L, 0.3 L, 0.5 L$ & $0.3 L$ & $(1 / 3) L$ \\
Test 2.0 & $0.3 L$ & $0.5 L$ to $2.0 L^{\mathrm{b}}$ & $1.0 L$ \\
Test 2.1 & $0.1 L$ & $0.5 L, 1.0 L, 1.5 L, 1.75 L$ & $1.0 L$ \\
Test 2.2 & $0.2 L$ & $0.5 L, 1.0 L$ & $1.0 L$ \\
Test 2.3 & $0.5 L$ & $0.5 L, 0.75 L, 1.0 L$ & $1.0 L$ \\
Test 3 & $0.15 L$ & $0.0 L, 0.5 L, 0.75 L, 1.0 L$ & $0.5 L$ \\
\hline
\end{tabular}

a Increments of $0.1 \mathrm{~L}$.

b Increments of $0.25 \mathrm{~L}$. 

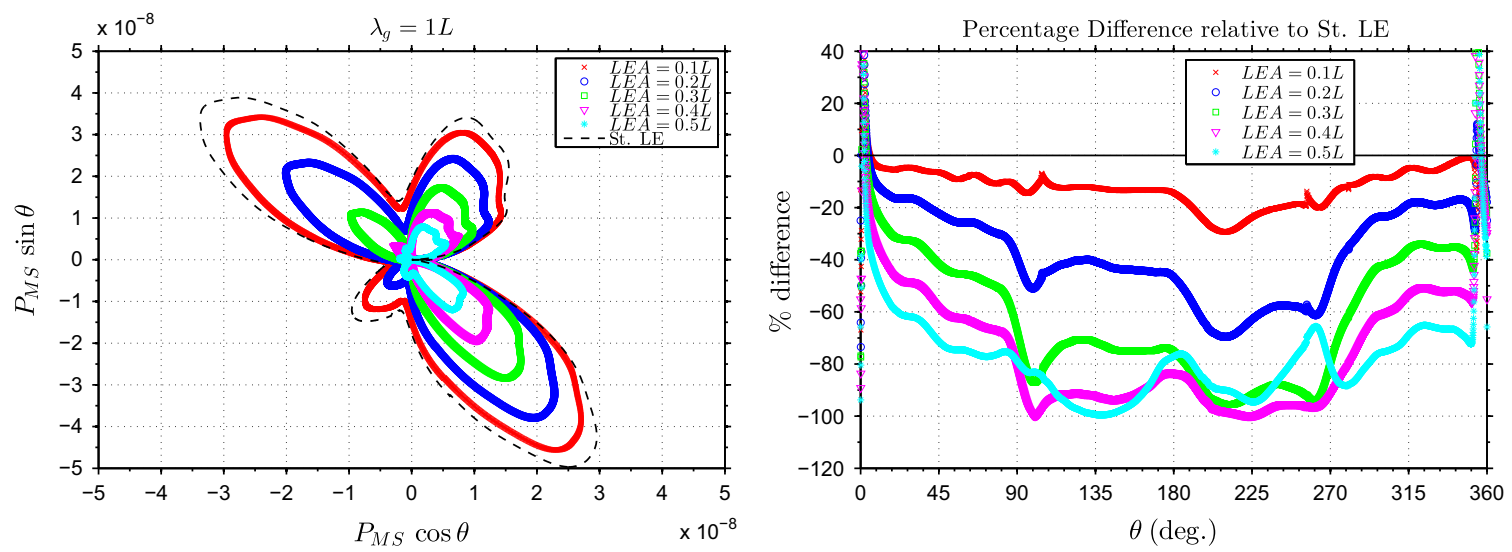

Fig. 11. Results of Test 1.0 for the effect of LEA based on a low-frequency gust.
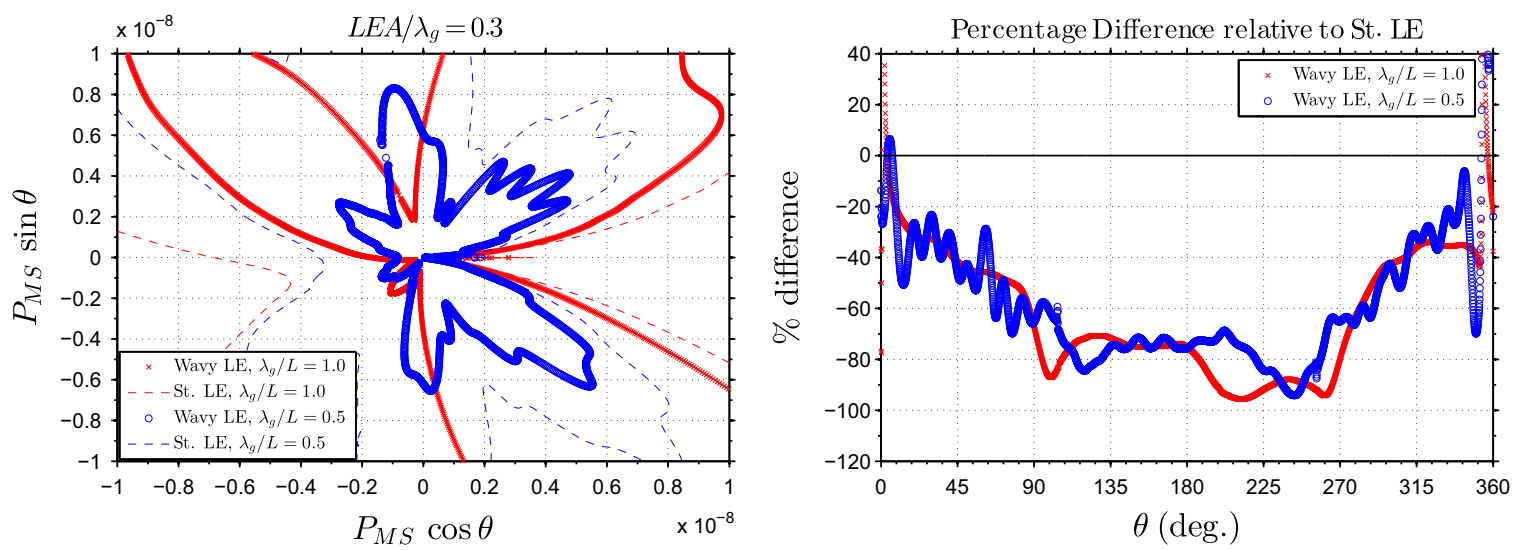

Fig. 12. The effect of LEA/ $\lambda_{g}$ : comparison of low- and medium-frequency gust cases.

LEA increases and the amount of reduction is particularly significant at the major angles of propagation. It is shown that 80 percent or higher percentage reduction in a wide range of angles can be achieved with LEA $=0.5 L$. However, there should be a certain limitation to the peak-to-peak LEA value in practical wind-turbine applications due to structural concerns.

Fig. 12 shows the result of additional tests with $L E A=0.15 \mathrm{~L}$ and $\mathrm{LEW}=0.5 \mathrm{~L}$ against the medium-frequency gust. The result of Test 1.0 with $\mathrm{LEA}=0.3 \mathrm{~L}$ is also shown together. For both tests, the ratio of wavy leading-edge amplitude to the longitudinal (streamwise) wavelength of the gust, denoted by LEA $/ \lambda_{g}$, is fixed at 0.3 . From Fig. 12, it can be seen that, although the incident gusts and the leading-edge geometries of the two tests are significantly different, the profiles of the relative (normalized) reduction of AGI noise look remarkably similar. This suggests that LEA $/ \lambda_{g}$ is the key factor that characterizes the performance of WLEs.

Fig. 12 also shows that the medium-frequency gust generates a smaller level of AGI noise and more complex directivity patterns with distinct lobes, compared to the low-frequency gust. Note that the amplitude parameters of the low- and the medium-frequency gusts are identical. Myers and Kerschen [3] analytically predicted for a 2D flat plate that the acoustic power of AGI noise would be inversely proportional to the reduced frequency of the gust.

Fig. 13 shows the results of Test 1.1 based on the high-frequency gust, which show cases that a high percentage reduction of AGI noise ( 80 percent or more) uniformly in all directions may be achieved when LEA/ $\lambda_{g}$ is sufficiently larger than 0.3 at the given LEW. This test also suggests that the amount of noise reduction may become saturated when LEA/ $\lambda_{g}$ is around 0.9. Note that the local data in the trailing-edge direction $\left(\theta=0^{\circ}\right.$ and $\left.360^{\circ}\right)$ may not be sufficiently meaningful since the original values of $P_{\mathrm{MS}}$ in that region are extremely small and numerical errors associated with the geometric singularity of the trailing-edge may have prevailed.

Fig. 14 compares the three different cases from the low-, medium- and high-frequency gusts with the same value of $\mathrm{LEA} / \lambda_{\mathrm{g}}=0.3$. The similarity in the profiles of relative (normalized) noise reduction at the same value of LEA $/ \lambda_{g}$, which was conjectured earlier from Fig. 12, now looks more convincing. This confirms that LEA/ $\lambda_{g}$ is the key factor to predict and characterize the aeroacoustic performance of WLEs for the reduction of AGI noise. 

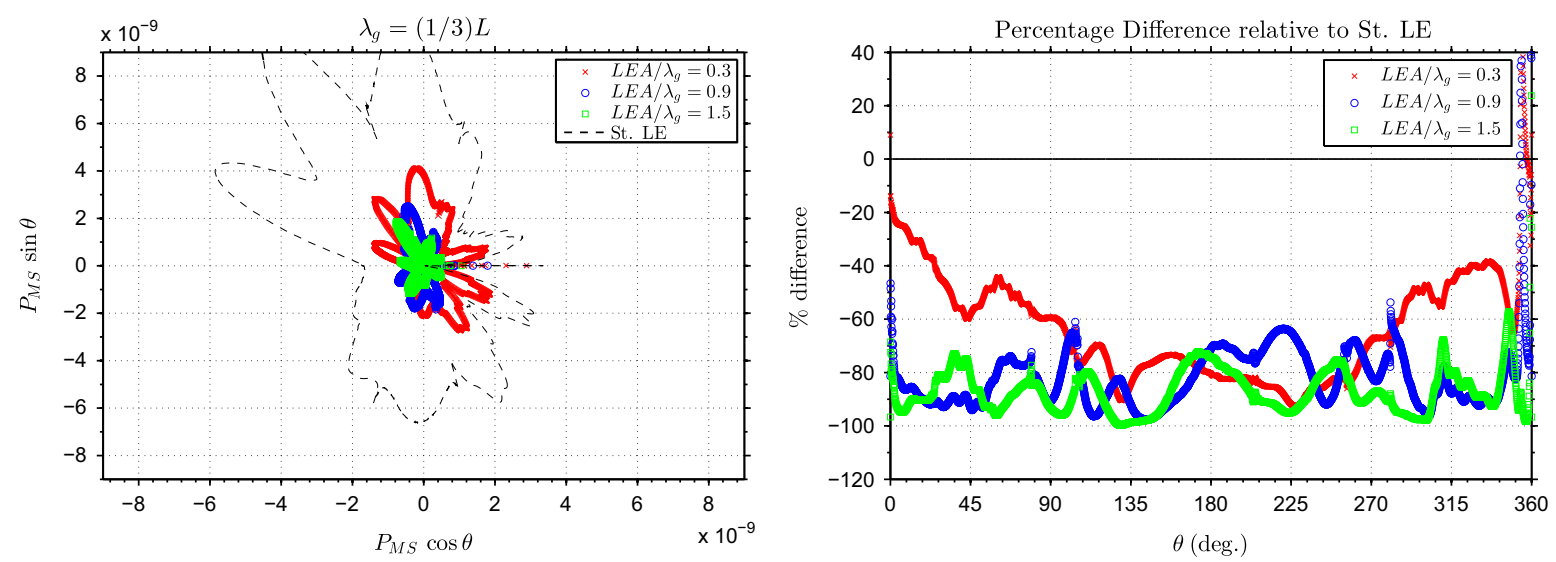

Fig. 13. Results of Test 1.1 for the effect of LEA/ $\lambda_{g}$ based on a high-frequency gust.
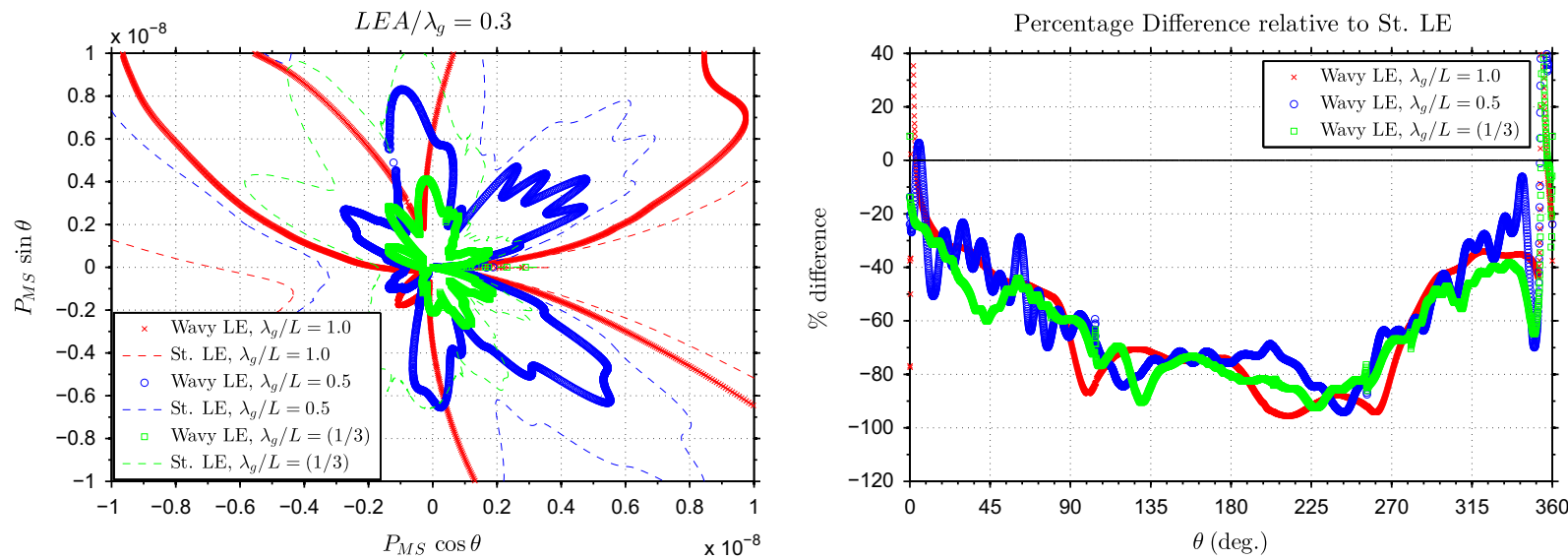

Fig. 14. The effect of LEA/ $\lambda_{g}$ : comparison of low-, medium- and high-frequency gust cases.
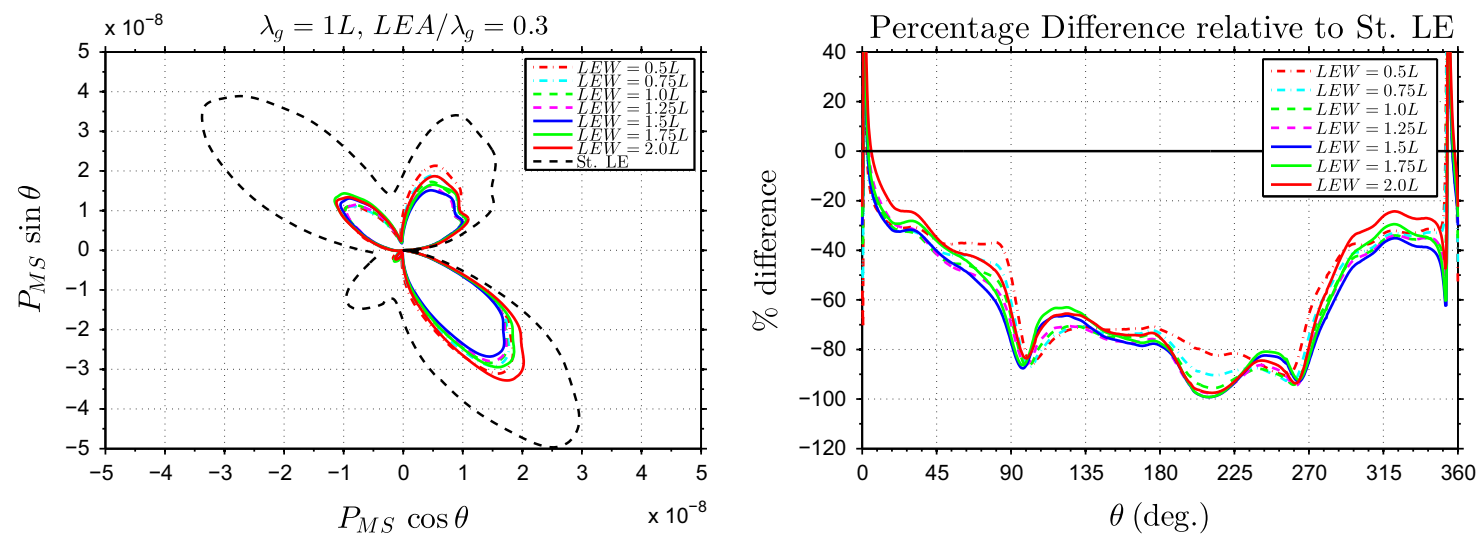

Fig. 15. Results of Test 2.0 for the effect of LEW.

\subsection{The effect of LEW}

Fig. 15 shows the directivity patterns of noise propagation and the profiles of relative noise reduction for Test 2.0 where various wavelengths of the leading edge are investigated. In this test, LEA $/ \lambda_{g}$ is kept at 0.3 and, again, the similarity in the profiles of relative noise reduction takes place irrespective of various LEWs. Although the profiles show insignificant differences against each other due to the similarity prevailing, the results suggest that some improvement in noise reduction may be achieved by adjusting LEW. 
To further investigate the effect of LEW, Tests 2.1, 2.2 and 2.3 are conducted for three different values of LEA/ $\lambda_{g}$ and the results are plotted in Figs. 16, 17 and 18, respectively. Overall, the results show the major dependency of LEA/ $\lambda_{\mathrm{g}}$ with minor variations against LEW. It is conjectured that the minor effect of LEW might be attributed to the present gust model containing insignificant spanwise disturbances, which results in weak interactions with the leading edges. In Fig. 16 for $\mathrm{LEA} / \lambda_{\mathrm{g}}=0.1$, it can be seen that there is an additional reduction in noise between $\mathrm{LEW} / \lambda_{\mathrm{g}}=0.5$ and 1.0 . The noise reduction seems to reach the maximum at $\mathrm{LEW} / \lambda_{\mathrm{g}}=1.5$ (as hinted in Test 2.0) but returns to the initial level at LEW $/ \lambda_{\mathrm{g}}=1.75$. The meaningful improvement of noise reduction taking place between LEW $/ \lambda_{g}=0.5$ and 1.0 is also exhibited in the following
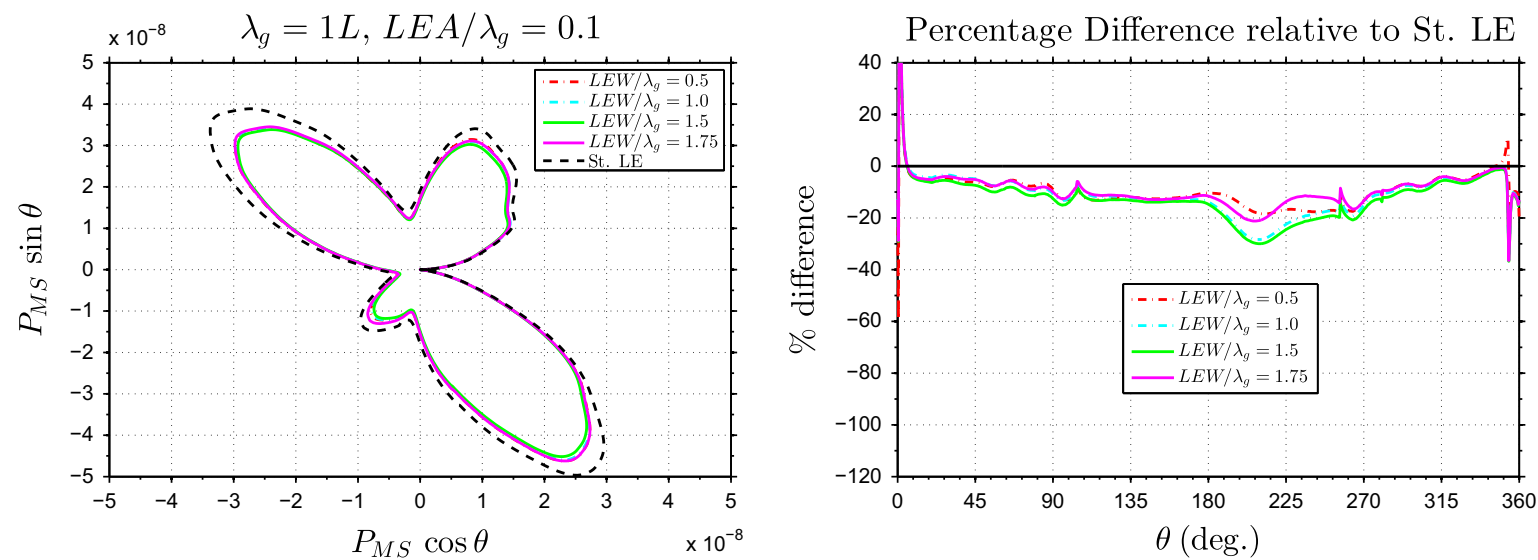

Fig. 16. Results of Test 2.1 for the effect of LEW.
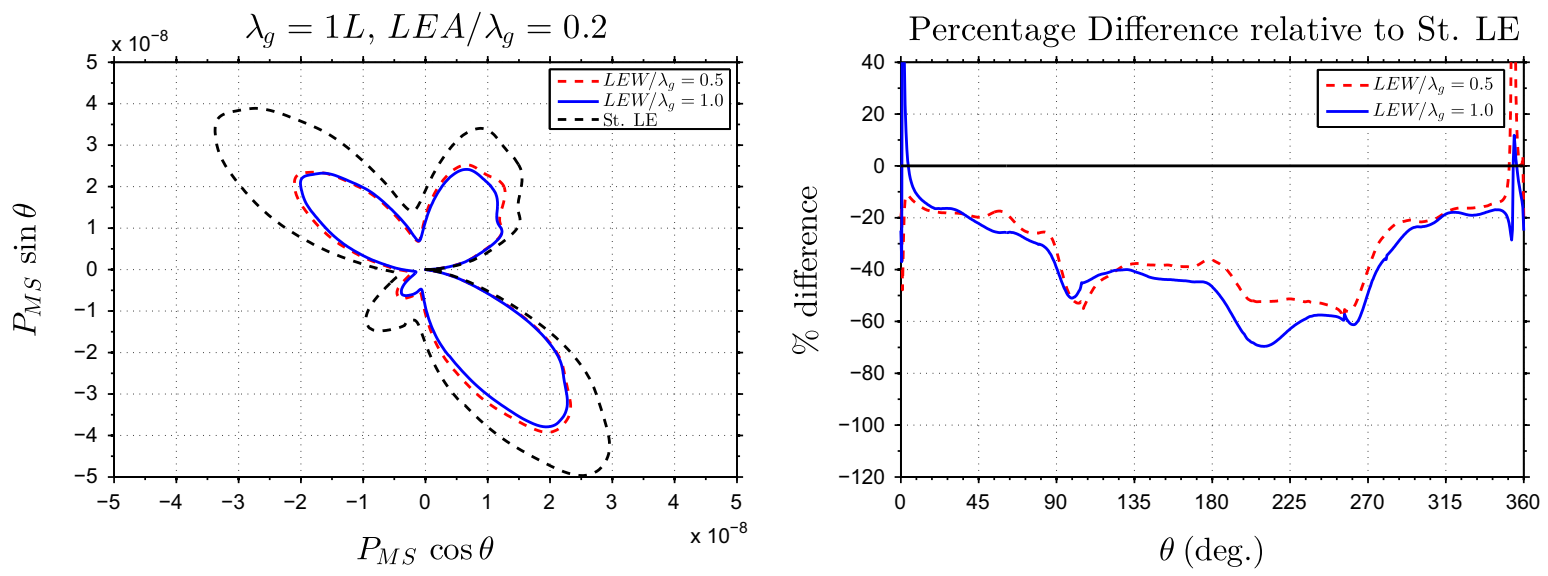

Fig. 17. Results of Test 2.2 for the effect of LEW.

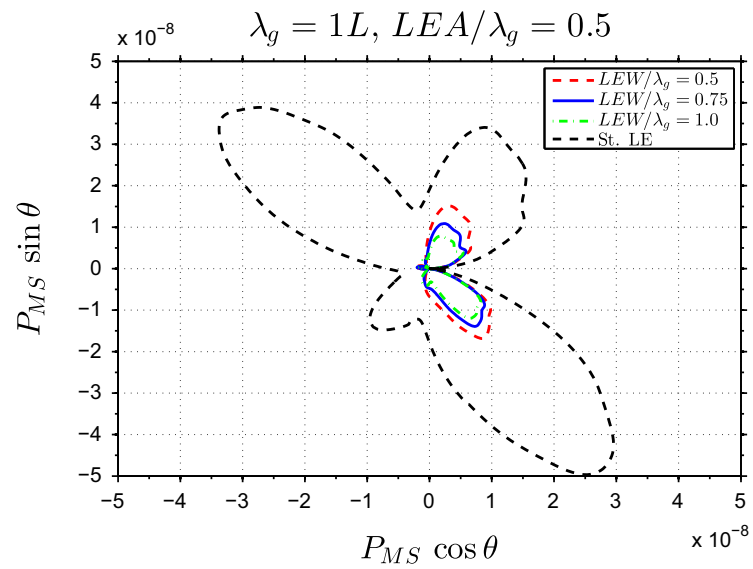

Percentage Difference relative to St. LE

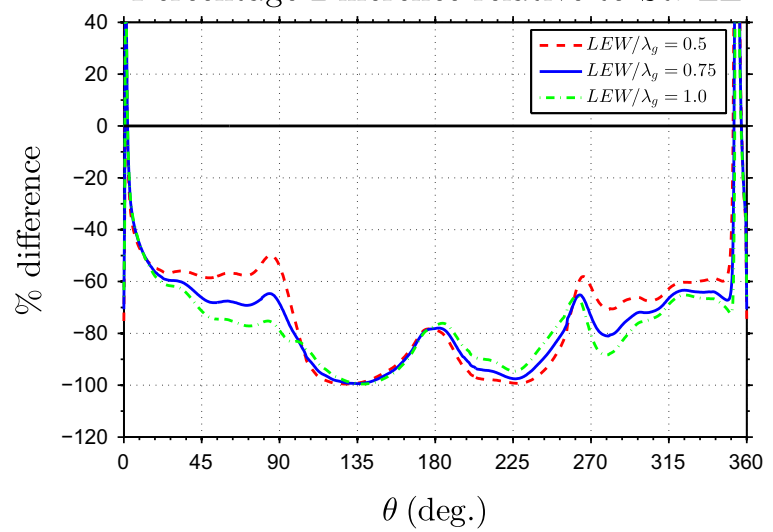

Fig. 18. Results of Test 2.3 for the effect of LEW. 

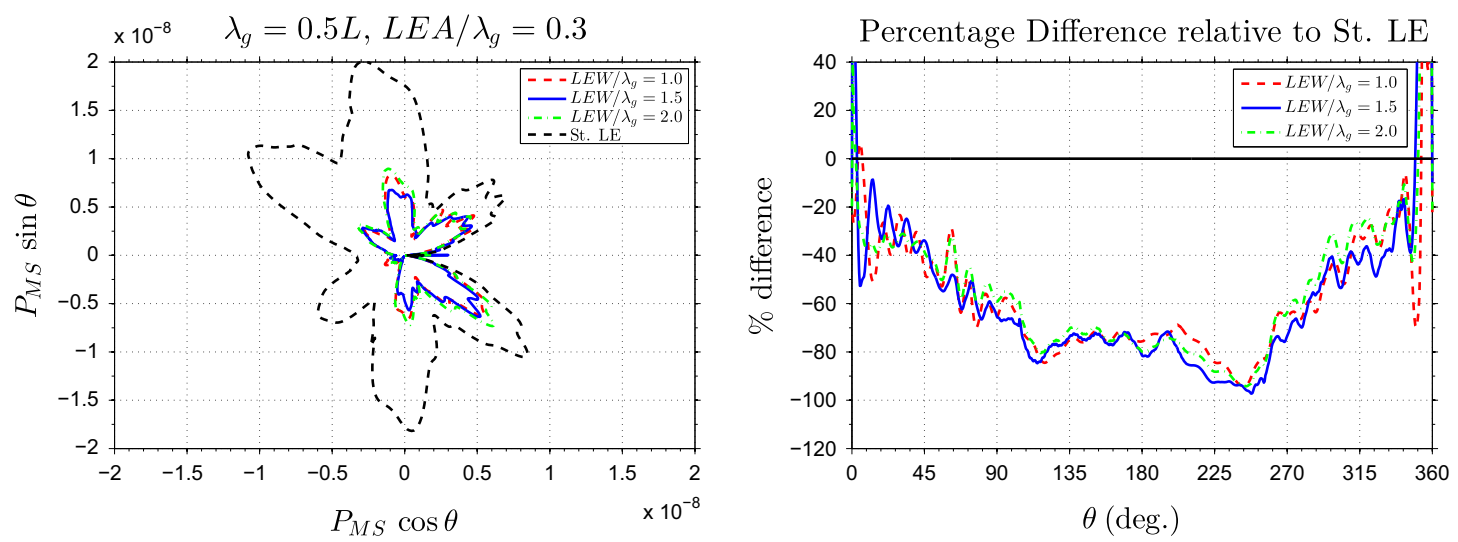

Fig. 19. Results of Test 3 for the effect of $\mathrm{LEW} / \lambda_{g}$.

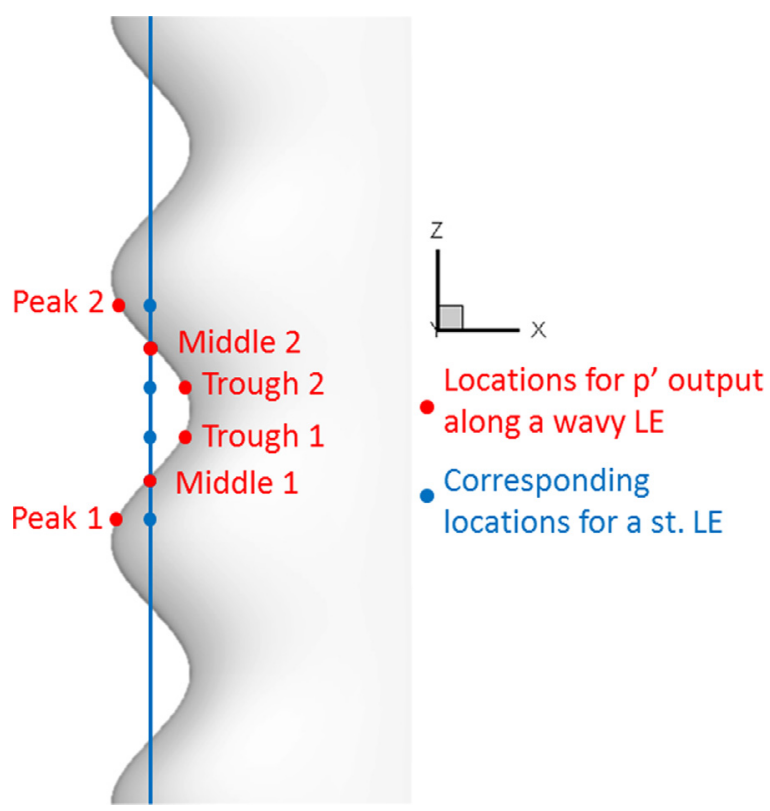

Fig. 20. Six transducer locations for the measurement of pressure fluctuations.

Figs. 17 and 18 for different values of LEA/ $\lambda_{g}=0.2$ and 0.5 . While these results are based on the low-frequency gust, Test 3 is carried out for the medium-frequency gust. Fig. 19 shows the results of Test 3 where LEA/ $\lambda_{g}$ is set to 0.3 and three different cases of $\mathrm{LEW} / \lambda_{\mathrm{g}}=1.0,1.5$ and 2.0 are compared. It can be seen that the same trends found in the low-frequency gust case still apply in the higher-frequency case. It is worth noting again that the effect of leading-edge wavelength (albeit less significant than LEA) becomes meaningful when $1.0 \leq \mathrm{LEW} / \lambda_{\mathrm{g}} \leq 1.5$ whereas the effect diminishes outside the range.

\section{The noise reduction mechanism of WLEs}

In this section, the AGI-noise-reduction mechanism of WLE is investigated. Two different geometries (LEA $=0.3 \mathrm{~L}$ and $0.4 \mathrm{~L}$ with both $\mathrm{LEW}=1.0 \mathrm{~L}$ ) are employed and the results are compared against the straight leading-edge case. The incident gust is also imposed by using Eq. (4) with $k_{1}=k_{2}=2 \pi$ which sets the longitudinal wavelength of the gust to $\lambda_{\mathrm{g}}=1.0 \mathrm{~L}$. The time signals of pressure fluctuation and its rate of change are recorded at six transducer points located around the leading edges. The transducer locations are depicted in Fig. 20 and they are namely, Peak 1 and 2; Middle 1 and 2; and, Trough 1 and 2. Although the straight leading edge has no peak or trough, the same names of transducers are used to indicate the corresponding locations.

The time signals of pressure fluctuation and its rate of change calculated and collected at the six transducer locations for each leading-edge geometry are plotted in Figs. 21 and 22, respectively. The results show that, for the straight leading edge, the signals are completely in-phase and are almost identical as anticipated. In contrast, WLEs produce significant phase shifts between signals from different locations (although no difference is observed between transducer 1 and 2 ). It seems 

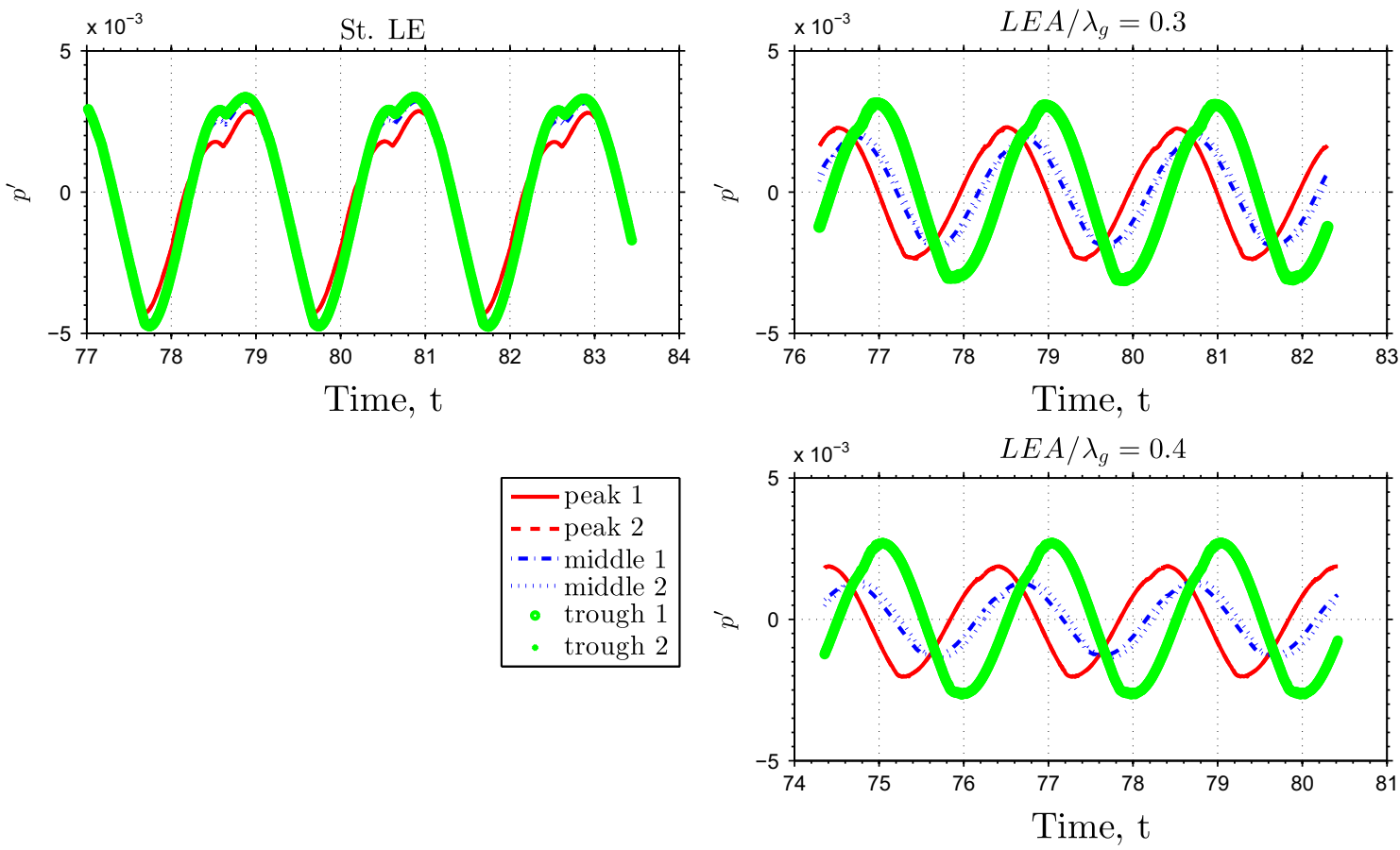

Fig. 21. Time signals of pressure fluctuation $\left(p^{\prime}\right)$ measured at the six transducer locations for three different leading-edge geometries.
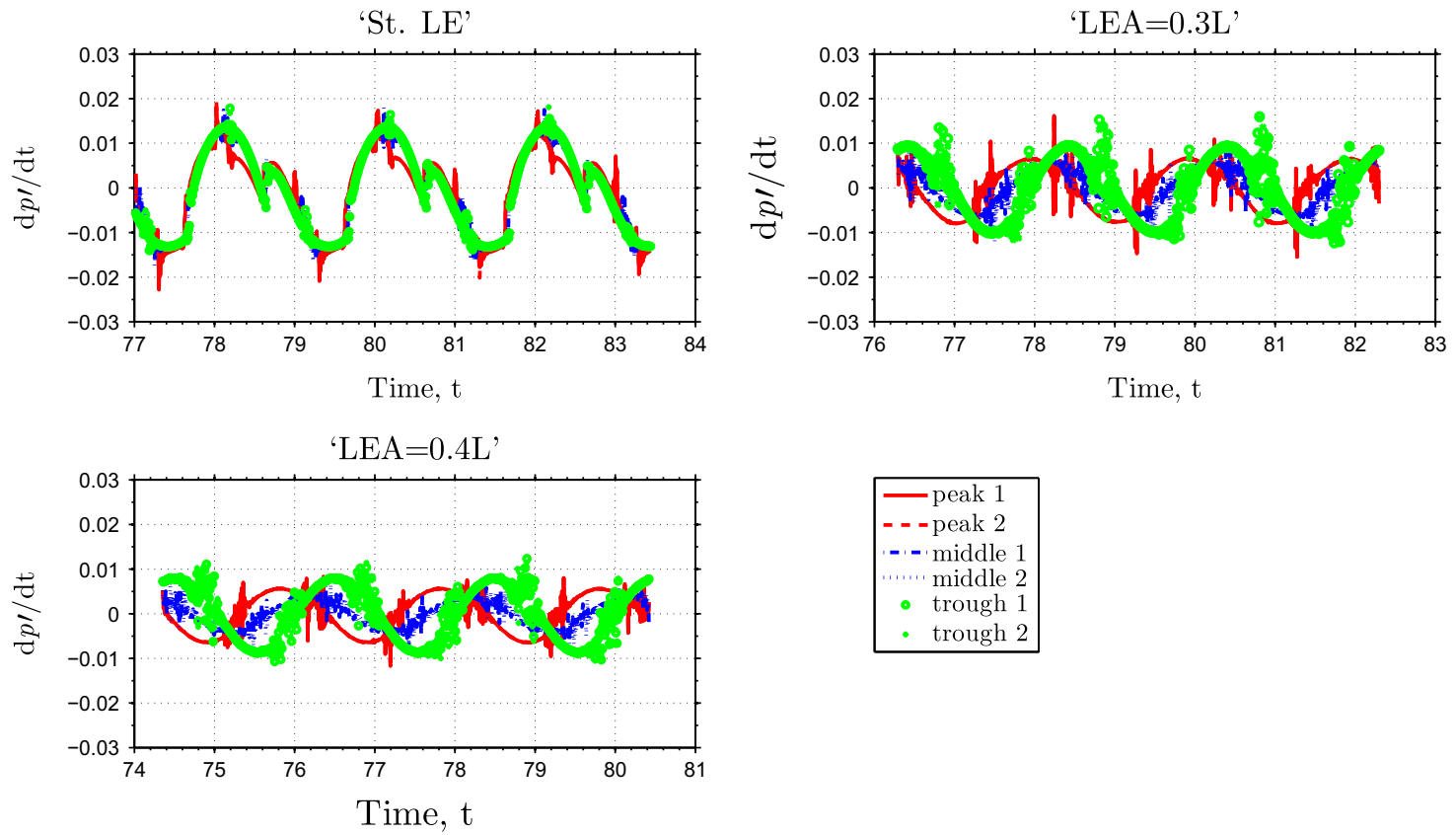

Fig. 22. Time signals of $\mathrm{d} p^{\prime} / \mathrm{d} t$ measured at the six transducer locations for three different leading-edge geometries.

apparent that the case of LEA $/ \lambda_{g}=0.4$ yields larger phase shifts and lower levels of fluctuations than the case of LEA $/ \lambda_{g}=$ 0.3. Both WLEs show noticeably decreased fluctuations compared to the straight leading edge.

In order to check the global effect of the WLEs, the pressure data are integrated over the aerofoil surface and the perturbed lift coefficient $\left(C_{L}^{\prime}\right)$ is calculated. The time signals of $C_{L}^{\prime}$ and $\mathrm{d} C_{L}^{\prime} / \mathrm{d} t$ for the three different leading edges are plotted in Fig. 23. It is confirmed that the WLEs reduce the amplitude of fluctuations compared to the straight leading-edge case and the reduction becomes more pronounced as LEA $/ \lambda_{g}$ increases.

The results indicate that the WLEs yield a de-synchronized gust response (phase shift) in span along the frontline of the aerofoil, which makes the local pressure fluctuations around the leading-edge area dispersed over the retarded period of 

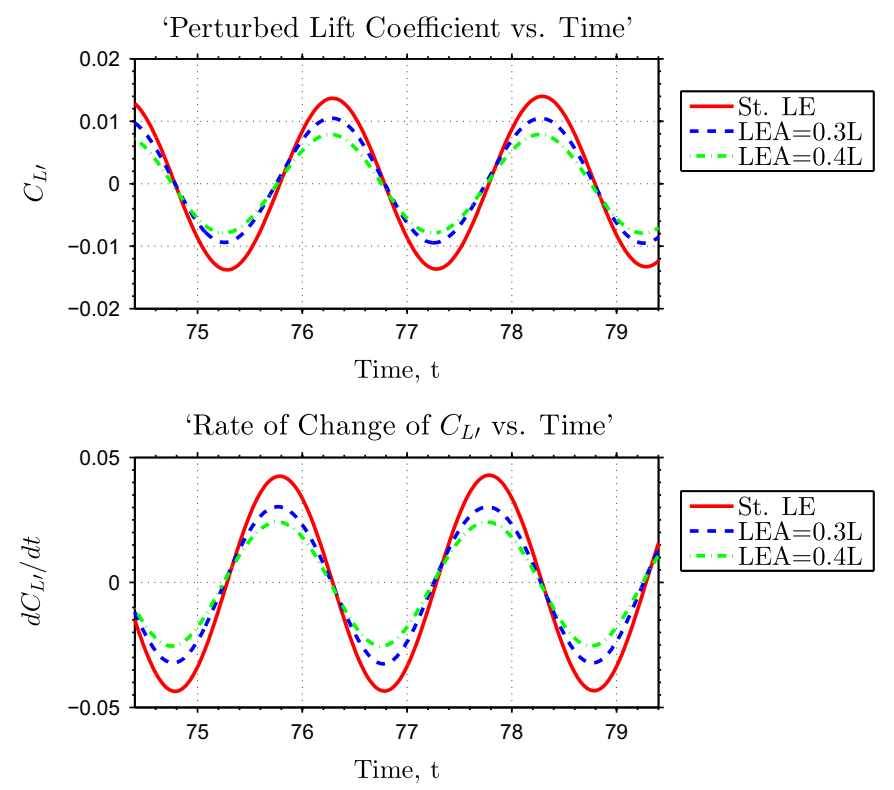

Fig. 23. $C_{L}^{\prime}$ time signals and rates of change for three different leading edges.

time. These local changes brought about by the WLEs in turn lead to a global reduction in the unsteady force acting on the aerofoil surface. The consequence of the reduced AGI noise can be explained by using the loading, or dipole, source term of the Ffowcs-Williams Hawkings (FW-H) equation. Farassat [36] have derived one of the solutions to the FW-H equation referred to as Formulation 1A. The far-field loading noise component of the Formulation 1A solution can be written as

$$
4 \pi p_{L}^{\prime}(\mathbf{x}, t)=\int_{l=0}\left[\frac{\left(\partial p^{\prime} / \partial \tau\right) \cos \theta}{\operatorname{cr}\left(1-M_{\mathrm{r}}\right)^{2}}\right]_{\text {source }} \mathrm{d} S,
$$

where $\tau$ is the source time (details can be found in [36]). This explains that the amplitude of $\mathrm{d} p^{\prime} / \mathrm{d} t$ at the source location and time is directly proportional to the magnitude of the propagated sound. Since the WLEs reduce the unsteady fluctuations both locally $\left(\mathrm{d} p^{\prime} / \mathrm{d} t\right)$ and globally $\left(\mathrm{d} C_{L}^{\prime} / \mathrm{d} t\right)$, the reduction of AGI noise is a direct consequence as described by the above formula.

\section{The effects of flow incidence angle and aerofoil thickness}

In this section, the effects of angle of attack $(\alpha)$ and aerofoil thickness associated with the WLEs on the reduction of AGI noise are studied. The same flow-gust condition as used in the previous section is maintained. The gust wavelength is also kept at $\lambda_{g}=1 L$. Three different angles of attack, $\alpha=1^{\circ}, 2^{\circ}$ and $3^{\circ}$, are tested. For this test, the NACA0015 aerofoil is used and the leading-edge parameters selected are LEA $/ \lambda_{g}=0.1$ and LEW $/ \lambda_{g}=0.5$. Fig. 24 shows the sound directivity patterns and the profiles of relative noise reduction varying with $\alpha$. It can be seen from the results that AGI noise level increases with $\alpha$ for both the straight and the WLE, although the overall shape of the directivity patterns seems unchanged. Myers and Kerschen [3] analytically predicted that the acoustic power of AGI noise (from a flat plate) increases almost linearly with the angle of attack in $0^{\circ} \leq \alpha \leq 3^{\circ}$ for $M_{\infty}=0.5$ (see Fig. 5 of [3]). In the meantime, the relative reduction of noise due to the WLEs remains more or less the same. This indicates that the noise reduction capability of WLEs is not significantly affected by the change of flow incidence angle (at least within $0^{\circ} \leq \alpha \leq 3^{\circ}$ ). An extended study on larger values of $\alpha$ would be useful but nonlinear viscous effects will need to be taken into account, which is beyond the scope of this paper.

For studying the effect of aerofoil thickness associated with WLEs, four different values of thickness: NACA0005, 0010, 0015 and 0020 are used. The same flow-gust condition and leading-edge geometry parameters used in the earlier study are maintained and $\alpha$ is kept at zero. The results are presented in Fig. 25. First, it can be seen that there exist remarkable changes in the sound directivity patterns varying with the aerofoil thickness. The main changes take place in the upper half-plane for this particular gust condition, and more sound propagates upstream as the aerofoil becomes thicker - these effects will need to be further investigated at a later publication. The second observation is that the profiles of relative noise reduction due to the WLEs (compared to the straight leading-edge case) still show a strong similarity at LEA $/ \lambda_{g}=0.1$ despite the substantial differences in thickness. 

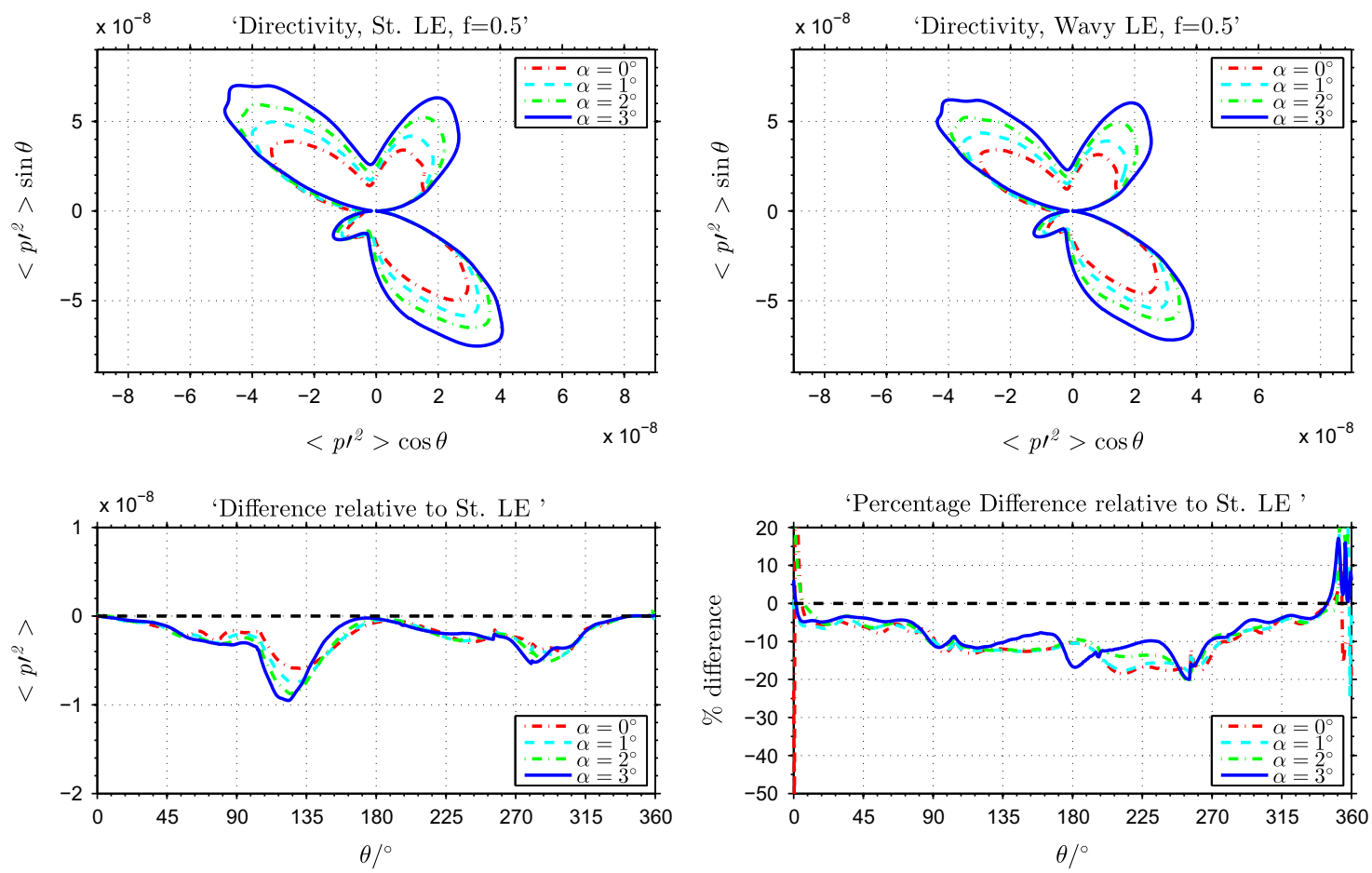

Fig. 24. The effect of various flow incidence angles on AGI noise: straight LE (left) and wavy LE (right) cases.
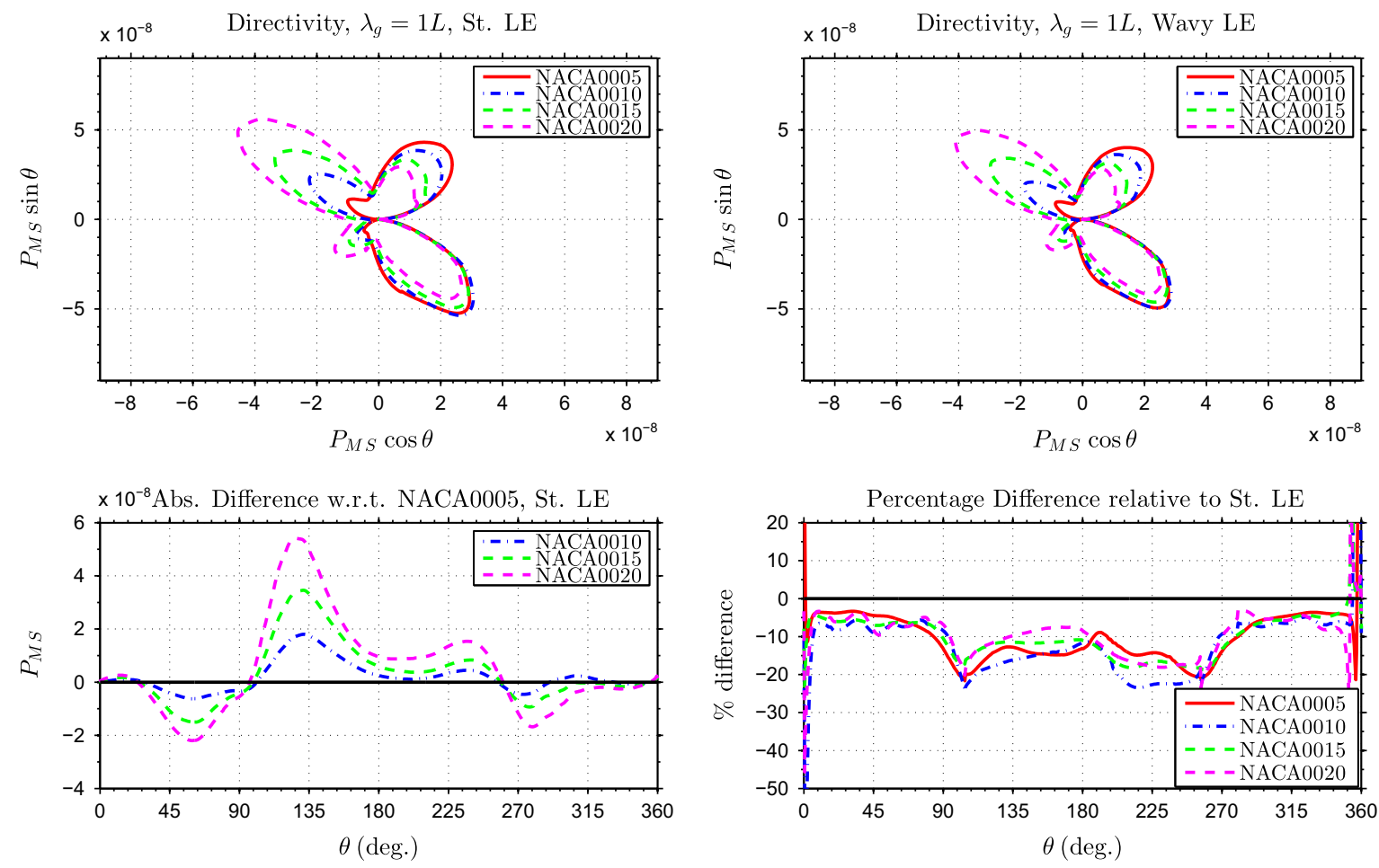

Fig. 25. The effect of aerofoil thickness: straight LE (left) and wavy LE (right) cases.

\section{The effects of multimode gusts}

In this section, incident flows with two constituent gust-modes are implemented to investigate their combined effects compared to the earlier single-mode cases. The results from this analysis may provide ground for further studies on how the 
wavy leading-edge geometry can be adjusted or optimized to reduce AGI noise effectively in a real atmospheric turbulence/ gust condition.

\subsection{Two-mode gust condition}

The present two-mode incident gust is specified by the parameters listed in Table 5 , where $f_{g}=M_{\infty} / \lambda_{g}$ denotes the gust frequency of each mode. The parameters are used for Eq. (4) to generate the velocity disturbances. The same amplitudes of disturbances are applied to both modes by using the same coefficients $A_{1}=B_{1}=0.005$ and $A_{2}=B_{2}=-0.004$, which is intended for comparison purposes. The NACA0015 aerofoil is used and two different WLEs named Wavy LE1 and Wavy LE2 with the same LEA but different LEW are employed, of which the geometric parameters are specified in Table 6. The ratios of the leading-edge parameters to the incident gust wavelengths in each mode are summarized in Table 7.

The results of the two-mode gust simulations are shown in Fig. 26. It is obvious that the two wavy leading-edge geometries lead to significantly reduced sound levels compared to the straight leading-edge case. However, the difference of noise reduction between the Wavy LE1 and Wavy LE2 cases is rather insignificant as it is discussed in Section 4.2 that LEW has a much less impact on noise reduction than LEA has. Most of the noise reduction comes from the effect of LEA as revealed in Section 4.1, and it is expected that the noise reduction is more effective against the second gust mode than the first one since the ratio of LEA $/ \lambda_{g}$ is higher with the second mode. To confirm this, sound pressure spectra are calculated at four different locations shown in Fig. 27, and the results are presented in Fig. 28. The two spectral peaks at $f_{g}=0.5$ and 0.75 correspond to the first and the second gust modes, respectively. It can be seen from Fig. 28 that both WLEs lead to significant

Table 5

Parameters for two constituent gust-modes used.

\begin{tabular}{llll}
\hline Mode & $\lambda_{g}$ & $f_{g}$ & $k_{1}=k_{2}$ \\
\hline 1 & $1.0 L$ & 0.50 & $2 \pi$ \\
2 & $(2 / 3) L$ & 0.75 & $3 \pi$ \\
\hline
\end{tabular}

Table 6

Wavy leading-edge parameters used for simulations with a two-mode gust.

\begin{tabular}{lcc}
\hline Name & LEA & LEW \\
\hline Wavy LE 1 & $0.3 L$ & $1.0 L$ \\
Wavy LE 2 & $0.3 L$ & $(2 / 3) L$ \\
\hline
\end{tabular}

Table 7

Parameters of two WLEs relative to each gust mode.

\begin{tabular}{llll}
\hline Mode & LEW $/ \lambda_{g}$ of Wavy LE 1 & LEW $/ \lambda_{g}$ of Wavy LE 2 & LEA $/ \lambda_{g}$ \\
\hline 1 & 1.0 & $(2 / 3) L$ & 0.30 \\
2 & 1.5 & 1.0 & 0.45 \\
\hline
\end{tabular}


Fig. 26. $P_{\mathrm{MS}}$ directivity patterns from two-mode gust simulations. 


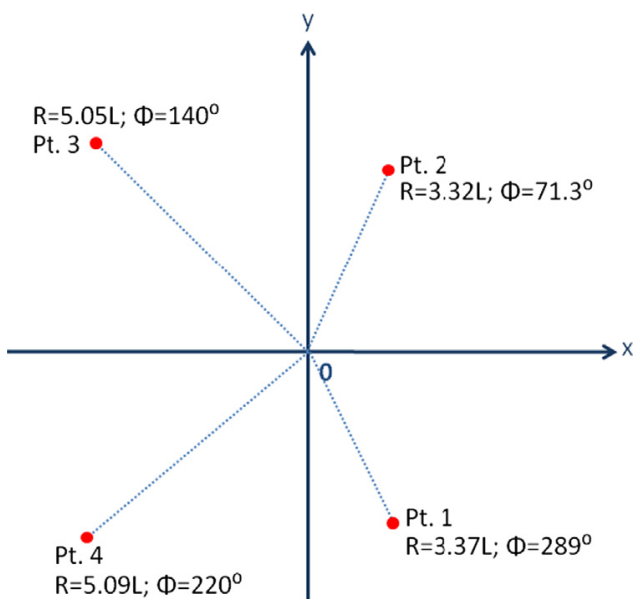

Fig. 27. Locations of observer points for the calculation of sound pressure spectra for the two-mode case.
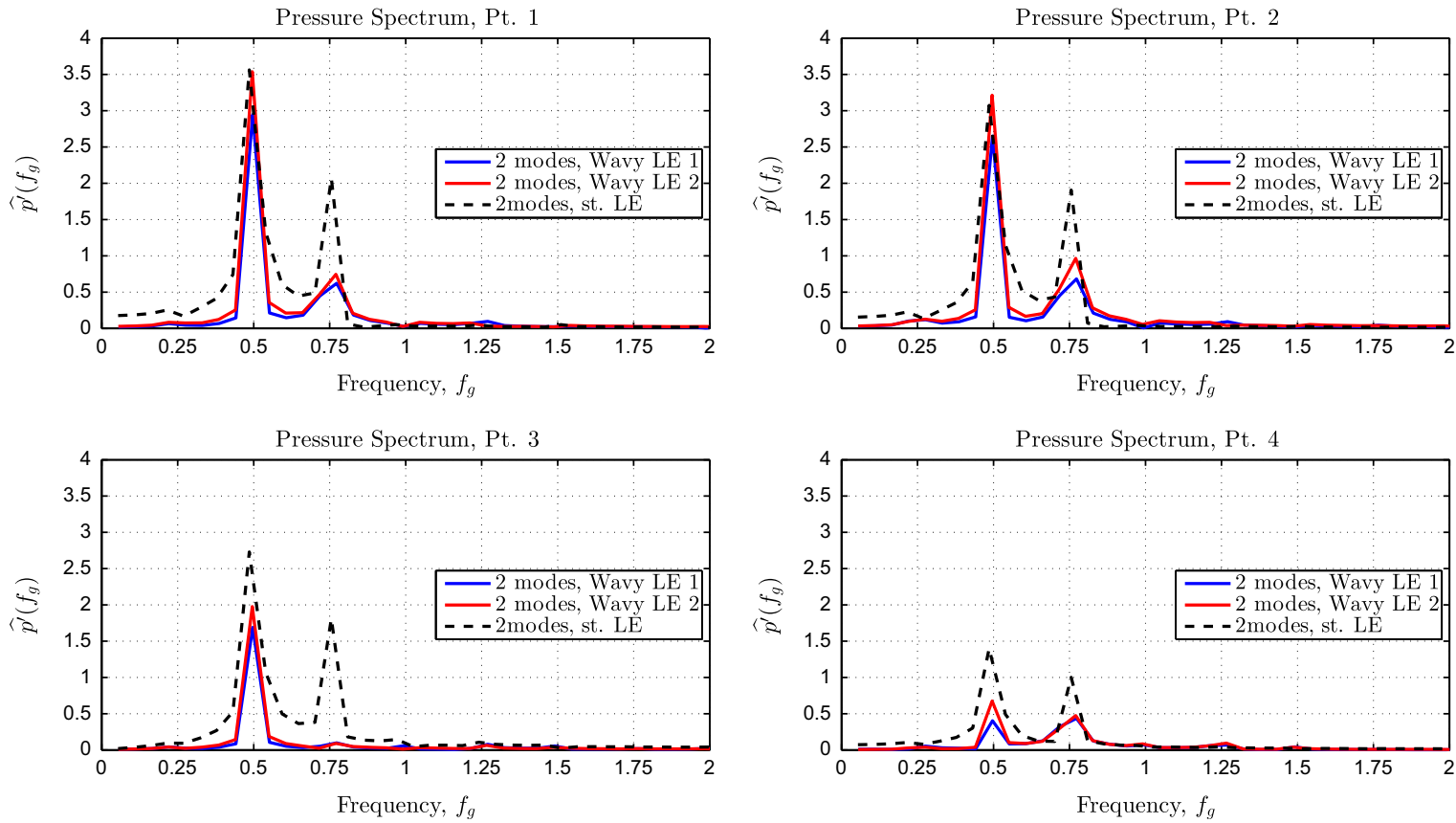

Fig. 28. Sound pressure spectra calculated at four different observer locations for the two-mode case.

noise reduction at the second mode frequency. Therefore, it is certain that LEA/ $\lambda_{g}$ plays the major role in AGI noise reduction against multimode gusts.

\section{Conclusions}

The present computational results show that the ratio of the peak-to-peak amplitude of WLEs to the longitudinal wavelength of incident gusts, denoted by LEA $/ \lambda_{g}$, is the most important factor to characterize the acoustic performance of WLEs in order to reduce AGI noise. The amount of noise reduction increases with LEA/ $\lambda_{\mathrm{g}}$ and saturates at around $\mathrm{LEA} / \lambda_{\mathrm{g}}=1.0$. It is found that the value of LEA $/ \lambda_{\mathrm{g}}$ at around 0.3 leads to a significant reduction of AGI noise up to 80 percent or more in a wide range of propagation angles (at a given wavelength of the incident gust). Also, it is conjectured that there exists a strong similarity rule that any two different profiles of relative (normalized) noise reduction (from different leadingedge geometries or incident gusts) match very well together when they have the same value of LEA/ $\lambda_{\mathrm{g}}$. In the meantime, the effect of LEW $/ \lambda_{g}$ turns out to be much less significant. This might be due to the fact that the spanwise velocity disturbances in the present gust model used were too weak to sufficiently interact with the leading-edge geometries. Nevertheless, it seems that there is a meaningful amount of extra noise reduction when $1.0 \leq \mathrm{LEW} / \lambda_{\mathrm{g}} \leq 1.5$ outside which the effect diminishes. A further study on the effects of $\mathrm{LEW} / \lambda_{g}$ will be required. 
The reduction mechanism of AGI noise using WLEs has been investigated. It is found that the WLEs induce a de-synchronized gust response (phase shift) in span along the frontline of the aerofoil, which makes the local pressure fluctuations around the leading-edge area dispersed over the retarded period of time. As a result, the dispersed pressure fluctuations at any spanwise location on a WLE have smaller amplitudes and time derivatives compared to those at the corresponding location on a straight leading edge. The attenuated level of pressure fluctuations on the aerofoil surface is the direct consequence of reduced dipole sound according to a well-known acoustic theory.

Further tests on the AGI-noise-reduction capability of WLEs have been carried out. The test results demonstrate that the relative amount of noise reduction is well maintained for various flow incidence angles and aerofoil thicknesses. The effectiveness of WLEs is also demonstrated through multimode gust tests. The multimode gust tests confirm that LEA/ $\lambda_{g}$ plays the major role in the noise reduction relative to the constituent gust wavelengths. For a given leading-edge wavelength (LEA), higher-frequency gust components are more effectively attenuated since the value of LEA/ $\lambda_{g}$ is larger. The present results provide a useful database for more practical applications of WLEs in the presence of a realistic atmospheric turbulence/gust.

\section{Acknowledgement}

The authors gratefully acknowledge that the present work (EP/J007633/1) has been supported by EPSRC (Engineering and Physical Sciences Research Council), Vestas Technology R\&D and SEEDA (South East England Development Agency). The authors also acknowledge the use of the IRIDIS high performance computing facility and associated support services at the University of Southampton in the completion of this work.

\section{References}

[1] P. Migliore, S. Oerlemans, Wind tunnel aeroacoustic tests of six airfoils for use on small wind turbines, Journal of Solar Energy Engineering 126 (4) (2004) 974-985.

[2] M.E. Goldstein, Unsteady vortical and entropic distortions of potential flows round arbitrary obstacles, Journal of Fluid Mechanics 89 (1978) $433-468$.

[3] M.R. Myers, E.J. Kerschen, Influence of incidence angle on sound generation by airfoils interacting with high-frequency gusts, Journal of Fluid Mechanics 292 (1995) 271-304.

[4] M.R. Myers, E.J. Kerschen, Influence of camber on sound generation by airfoils interacting with high-frequency gusts, Journal of Fluid Mechanics 353 (1997) 221-259.

[5] I. Evers, N. Peake, Noise generation by high-frequency gusts interacting with an airfoil in transonic flow, Journal of Fluid Mechanics 411 (2000) $91-130$.

[6] M.V. Lowson, A new prediction model for wind turbine noise, Renewable Energy International Conference on Clean Power, 1993, pp. 177-182.

[7] R.K. Amiet, Acoustic radiation from an airfoil in a turbulent stream, Journal of Sound and Vibration 41 (4) (1975) 407-420.

[8] J.R. Scott, H.M. Atassi, A finite-difference frequency-domain numerical scheme for the solution of the gust response problem, Journal of Computational Physics 119 (1) (1995) 75-93.

[9] D.P. Lockard, P.J. Morris, Radiated noise from airfoils in realistic mean flows, AIAA Journal 36 (6) (1998) $907-914$.

[10] V.V. Golubev, R.R. Mankbadi, R. Hixon, Space-time mapping analysis of airfoil nonlinear interaction with unsteady inviscid flow, AIAA Journal 43 (10) (2005) 2147-2156.

[11] R. Hixon, V.V. Golubev, R.R. Mankbadi, J.R. Scott, S. Sawyer, M. Nallasamy, Application of a nonlinear computational aeroacoustics code to the gustairfoil problem, AIAA Journal 44 (2) (2006) 323-328.

[12] H.M. Atassi, A.A. Ali, O. Atassi, I. Vinogradov, Scattering of incident disturbances by an annular cascade in a swirling flow, Journal of Fluid Mechanics 499 (2004) 111-138.

[13] R. Hixon, A. Sescu, S. Sawyer, Vortical gust boundary condition for realistic rotor wake/stator interaction noise prediction using computational aeroacoustics, Journal of Sound and Vibration 330 (2011) 3801-3817.

[14] J.W. Kim, A.S.H. Lau, N.D. Sandham, Proposed boundary conditions for gust-airfoil interaction noise, AIAA Journal of Technical Notes 48 (11) (2010) $2705-2710$.

[15] P.T. Soderman, Leading Edge Serrations which Reduce the Noise of Low-speed Rotors, Technical Report, NASA, 1973.

[16] S. Ito, Aerodynamic influence of leading-edge serrations on an airfoil in a low Reynolds number-a study of an owl wing with leading edge serrations, Journal of Biomechanical Science and Engineering 4 (1) (2009) 117-123.

[17] D.S. Miklosovic, M.M. Murray, L.E. Howle, F.E. Fish, Leading-edge tubercles delay stall on humpback whale (Megaptera novaeangliae) flippers, Physics of Fluids 16 (5) (2004) L39-L42.

[18] K.L. Hansen, R.M. Kelso, B.B. Dally, Performance variations of leading-edge tubercles for distinct airfoil profiles, AIAA Journal 49 (1) (2011) $185-194$.

[19] F.E. Fish, J.M. Battle, Hydrodynamic design of the humpback whale flipper, Journal of Morphology 225 (1) (1995) 51-60.

[20] F.E. Fish, L.E. Howle, M.M. Murray, Hydrodynamic flow control in marine mammals, Integrative and Comparative Biology 48 (6) (2008) $788-800$.

[21] H. Johari, C. Henoch, D. Custodio, A. Levshin, Effects of leading-edge protuberances on airfoil performance, AIAA Journal 45 (11) (2007) $2634-2642$.

[22] M.J. Stanway, Hydrodynamic Effects of Leading-edge Tubercles on Control Surfaces and in Flapping Foil Propulsion, Master's Thesis, Massachusetts Institute of Technology, 2008

[23] D. Custodio, The Effect of Humpback Whale-like Leading Edge Protuberances on Hydrofoil Performance, Master's Thesis, Worcester Polytechnic Institute, 2007.

[24] H.T.C. Pedro, M.H. Kobayashi, Numerical study of stall delay on humpback whale flippers, 46th AIAA Aerospace Sciences Meeting and Exhibit, 2008

[25] H.S. Yoon, P.A. Hung, J.H. Jung, M.C. Kim, Effect of the wavy leading edge on hydrodynamic characteristics for flow around low aspect ratio wing, Computers \& Fluids 49 (1) (2011) 276-289.

[26] K.L. Hansen, R.M. Kelso, C.J. Doolan, Reduction of flow induced tonal noise through leading edge tubercle modifications, 16th AIAA/CEAS Aeroacoustics Conference Proceedings, 2010.

[27] J.W. Kim, D.J. Lee, Generalized characteristic boundary conditions for computational aeroacoustics, AIAA Journal 38 (11) (2000) $2040-2049$.

[28] J.W. Kim, D.J. Lee, Generalized characteristic boundary conditions for computational aeroacoustics part 2, AIAA Journal 42 (1) (2003) 47-55.

[29] J.W. Kim, Optimised boundary compact finite difference schemes for computational aeroacoustics, Journal of Computational Physics 225 (1) (2007) 995-1019.

[30] J.W. Kim, High-order compact filters with variable cut-off wavenumber and stable boundary treatment, Computers \& Fluids 39 (7) (2010) 1168-1182.

[31] S. Haeri, J.S. Shrimpton, Cfdcomm: an optimized library for scalable point-to-point communication for general cfd applications, High Performance Computing and Communication 2012 IEEE 9th International Conference on Embedded Software and Systems (HPCC-ICESS), 2012, pp. $1001-1006$. 
[32] J.W. Kim, R.D. Sandberg, Efficient parallel computing with a compact finite difference scheme, Computers E Fluids 58 (2012) $70-87$.

[33] M.D. Dahl (Ed.), Third Computational Aeroacoustics (CAA) Workshop on Benchmark Problems, Technical Report NASA/CP-2000-209790, NASA, 2000.

[34] M.D. Dahl (Ed.), Fourth Computational Aeroacoustics (CAA) Workshop on Benchmark Problems, Technical Report NASA/CP-2004-212954, NASA, 2004.

[35] R.E. Sheldahl, P.C. Klimas, Aerodynamic Characteristics of Seven Symmetrical Airfoil Sections through 180-degree Angle of Attack for use in Aerodynamic Analysis of Vertical Axis Wind Turbines, Technical Report, Sandia National Laboratories, 1981.

[36] F. Farassat, Derivation of Formulations 1 and 1A of Farassat, Technical Report, NASA, 2007. 